\title{
Thermodynamic Gross-Neveu model in a constant electromagnetic field
}

\author{
Shinya Kanemura ${ }^{a)}$ 巴 Haru-Tada Sato ${ }^{b)}$ \& and Hiroshi Tochimura ${ }^{c)}$ 队 \\ a) Theory Group, KEK, \\ Tsukuba, Ibaraki 305, Japan, \\ b) The Niels Bohr Institute, University of Copenhagen, \\ Blegdamsvej 17, DK-2100 Copenhagen, Denmark \\ c) Department of Physics, Hiroshima University, \\ Kagamiyama 1-3-1, Higashi-Hiroshima 739, Japan
}

\begin{abstract}
Analyzed is the effective potential of $D$-dimensional thermodynamic Gross-Neveu model (defined by large- $N$ leading order, $2 \leq D<4$ ) under constant electromagnetic field $(\vec{E} \cdot \vec{B}=0)$. The potential is derived from a thermal analogy of the worldline formalism. In the magnetic case, the potential is expressed in terms of a discretized momentum sum instead of a continuum momentum integral, and it reproduces known critical values of $\mu$ and $T$ in the continuum limit. In the electric case, chiral symmetry is restored at finite $T$ (with arbitrary $\mu$ ) against instability of fermion vacuum. An electromagnetic duality is pointed out as well. Phase diagrams are obtained in both cases.
\end{abstract}

PACS: 11.30.Rd, 12.20.Ds

Keywords: Gross-Neveu model, worldline formalism, effective potential

\footnotetext{
${ }^{1}$ e-mail: kanemu@theory.kek.jp

${ }^{2}$ e-mail: sato@nbi.dk

${ }^{3}$ e-mail: tochi@theo.phys.sci.hiroshima-u.ac.jp
} 


\section{Introduction}

The Gross-Neveu (GN) model is known as the simplest model possessing the similar properties to QCD; dynamical breaking of (discrete) chiral symmetry, dimensional transmutation, asymptotic freedom and renormalizability $(D<4)$. Indeed, a number of GN models (and related models such as the NJL models) have often been studied as the prototypes of dynamical symmetry breaking in the various circumstances of finite temperature [1, 18, 19], electromagnetic field [2] -11] and finite curvature [15, 16]. The high temperature behaviors of these models [7, 11, 14, 17] may potentially be interesting for exploring a dynamical symmetry breaking in (quark-gluon) plasma and in cosmological situations like early universe or a neutron star.

In this paper, we examine the effective potential and chiral phase structures of the $D$-dimensional $(2 \leq D<4)$ GN model defined by the leading order of $1 / N$-expansion in a magnetic $B$ (or electric $E$ ) background field at finite temperature and density. In the case of $D$ being slightly below 4, the plasma physics is an interesting application [14]. In the case of $D=3$ [1], the physics of our model concerns planar condensed matter systems such as BCS theory: a magnetic field works as a catalyst for generating an energy gap in fermion spectra, through enhancing the interactions of fermions at small momenta even if the weakest (attractive) interaction. Gusynin et. al. suggested [11, 12] that this effect should be universal, i.e., model independent, in $D=3$ and should be considered in the phenomena such as quantum Hall effects and high temperature superconductivity. Similarly in a supersymmetric model, constant magnetic field generates fermion's dynamical mass 13.

In the case of $D=2$, magnetic field can not exist, however the GN model by itself corresponds to a model of poly-acetylene. The first order phase transition of poly-acetylene from solitonic (broken) phase to metallic (symmetric) phase is formulated [20] as a function of doping concentration (chemical potential) within the large- $N$ approximation. The critical value of chemical potential coincides with an experimental value, and recently a $1 / N$-correction to this result was obtained in [21]. Our study might explain some thermodynamic behaviour of a poly-acetylene model under constant electric field in a finite temperature and density system. 
Our crucial interest is therefore the question how universal the effect of magnetic catalyst on phase structures is in the setting of both finite temperature $T$ and chemical potential $\mu$ in various dimensions. We study this question for an electric background field as well. In order to deal with these models all together, it is, of course, useful to analyze a dimensionally regulated effective potential. Such approaches were done in the cases of finite temperature [19], curvature [15] and their mixture [17. We shall derive a universal expression of the effective potential as a function of $D, T, \mu$ and the external field parameter $\xi\left(=e \sqrt{|\vec{B}|^{2}-|\vec{E}|^{2}}\right.$, where we have assumed $\left.\vec{B} \cdot \vec{E}=0\right)$ within the leading order of the $1 / N$-expansion. We shall then present various interesting properties which can be derived from the universal effective potential. Although we have not completed our analyses yet, we confirm that our present results will be useful in order to establish a foundation for further developments in the various branches mentioned above.

This paper is organized as follows. In Sect.2, we present a simple and convenient derivation of the effective potential. There is no manifestation of exact fermion propagators of finite temperature field theory in the derivation. Namely, we do not use its Feynman rules to evaluate a fermion loop. Indeed, we apply an analogy of the worldline formalism [22, 23] to the finite temperature cases. Then we write down the proper-time integral representations for the critical equations which determine the phase structures in the $\xi$ $T$ - $\mu$ space.

In Sect.3, we discuss the cases of magnetic field dominant, i.e., $\xi$ is a real number. In the former half of this section, we analyze the zero-temperature limit of the effective potentials for finite $\xi$ and $\mu$. The potential is shown to be a discretized version of the one presented in [19]. Also, first order phase transitions are observed in large $\mu$ regions on the $\xi-\mu(T=0)$ plane. In the latter half of the section, we discuss the phase structures. We find that a constant magnetic field works as the catalyst in $3 \leq D<4$ at finite $T$ and $\mu$, and verify that the magnitude of catalyst effect becomes smaller as $D$ or $\mu$ increasing.

In Sect.4, we present the electric dominant phase diagrams, i.e., the cases of $\xi$ a pure imaginary number. Analyzing the imaginary part of the effective potential, we show that chiral symmetry can be restored in high $T$ situations $(\mu \geq 0)$ in $2 \leq D<4$, with vanishing imaginary part. We also show that some analytic results at $T=\mu=0$ can be obtained 
through the electromagnetic dual rotation $\xi \rightarrow i \rho$.

Conclusions and discussions are in Sect.5. In Appendices, some details on the $\xi \rightarrow 0$ limit are presented. One can find how to reproduce various known results at $\xi=0$ [18, 20, 19] from our results in this continuum limit $\xi \rightarrow 0$.

\section{The effective potential in $\xi-T-\mu$ space}

The model Lagrangian is

$$
\mathcal{L}=\bar{\psi} i \gamma^{\mu} D_{\mu} \psi-\frac{N}{2 \lambda} \sigma^{2}-\sigma \bar{\psi} \psi
$$

where $\sigma$ is the auxiliary field and $N$ the number of flavors. The fermions $\psi=\left\{\psi_{i}\right\},(i=$ $1, \cdots, N)$, are coupled to the background gauge fields via the covariant derivative $D_{\mu}=$ $\partial_{\mu}-i e A_{\mu}$. The effective potential is given by

$$
V(\sigma, \xi)=V(\sigma)-V(0)
$$

with

$$
V(\sigma)=\frac{1}{2 \lambda} \sigma^{2}-\ln \operatorname{Det}\left(i \gamma^{\mu} D_{\mu}-\sigma\right) .
$$

This model does not possess a continuum chiral symmetry but a discrete one. After the effective potential is obtained as a function of the auxiliary field, the potential becomes the same form as that of the NJL model.

We shall show below that the $D$-dimensional thermodynamic effective potential is given by

$$
V(\sigma ; \xi, \beta, \mu)=\frac{1}{2 \lambda} \sigma^{2}+\frac{\operatorname{tr}[1]}{2 \beta} \int_{0}^{\infty} \frac{d s}{s} \frac{\Theta_{2}\left(s \frac{2 \mu}{\beta}, i s \frac{4 \pi}{\beta^{2}}\right)}{(4 \pi s)^{(D-1) / 2}} s \xi \operatorname{coth}(s \xi) e^{s \mu^{2}}\left(e^{-s \sigma^{2}}-1\right),
$$

where $\Theta_{2}$ is the elliptic theta function of second kind and $\operatorname{tr}[1]$ means the trace of gamma matrix unit (we do not need its explicit value). The $D=3$ (magnetic dominant) case was derived in [11]. Also, by letting $T\left(=\beta^{-1}\right)$ and $\mu$ to be zero, this potential reproduces the potentials derived in the $D=3$ magnetic dominant (real $\xi$ ) cases [5, [] and the electric dominant (pure imaginary $\xi$ ) cases in $D=2$ [3, 25], $D=3$ [6] and $D=4$ [团. 


\subsection{Derivation of the effective potential}

In this subsection, we present a somewhat different derivation of the effective potential from that done in [11], where they evaluated it in the Matsubara (imaginary time) method using a fermion propagator under a constant magnetic field. We shall derive the potential (2.4), modifying the worldline formalism [22, 23] into the thermodynamic case.

Let us start with reviewing a few notes on $V(\sigma)$ for convenience of notation. In the Schwinger proper time method [26], a basic expression for the potential (2.3) already exists in [2]

$$
V(\sigma)=\frac{1}{2 \lambda} \sigma^{2}+\frac{1}{2} \operatorname{tr} \int_{0}^{\infty} \frac{d s}{s} \frac{1}{(4 \pi s)^{D / 2}} e^{-s \sigma^{2}} \exp \left[-\frac{1}{2} e s F_{\mu \nu} \sigma^{\mu \nu}-\frac{1}{2} \operatorname{Tr} \ln \left(\frac{\sin (e s F)}{e s F}\right)_{\mu \nu}\right]
$$

where tr and Tr mean the traces in the Gamma and Lorentz matrix spaces respectively. The trace of $F_{\mu \nu}^{2 n}$ is given by the following in respective dimensions: $2 E^{2 n}$ in $D=2$, $2\left(\vec{E}^{2}-B^{2}\right)^{n}$ in $D=3$ and $2\left[\left(\rho_{+}^{2}\right)^{n}+\left(\rho_{-}^{2}\right)^{n}\right]$ in $D=4$, where

$$
\rho_{ \pm}^{2}=\frac{1}{2}\left[\vec{E}^{2}-\vec{B}^{2} \pm \sqrt{\left(\vec{B}^{2}-\vec{E}^{2}\right)^{2}+4(\vec{E} \cdot \vec{B})^{2}}\right]
$$

If we assume $\vec{E} \cdot \vec{B}=0$ in $D=4$, and if the notation $\rho=e \sqrt{\vec{E}^{2}-\vec{B}^{2}}$ is properly understood in each case (for example, $\vec{B}$ should be zero in $D=2$ ), we can write those trace values in a unified form

$$
\operatorname{Tr}\left[(e F)^{2 n}\right]_{\mu \nu}=2 \rho^{2 n} .
$$

Similarly, the eigenvalue of $\frac{1}{2} e \sigma \cdot F$ can be expressed as $\pm i \rho$ multiplied by the degeneracy factor $\frac{1}{2} \operatorname{tr}[1]$ in each $D$, where we have again used $\vec{E} \cdot \vec{B}=0$ in $D=4$. In this way, we re-write

$$
\begin{aligned}
& \operatorname{tr} \exp \left[-\frac{1}{2} e s F_{\mu \nu} \sigma^{\mu \nu}\right]=\operatorname{tr}[1] \cosh (s \xi), \\
& \operatorname{Tr}\left[\ln \frac{\sin (e s F)}{e s F}\right]_{\mu \nu}=2 \ln \frac{\sinh (s \xi)}{s \xi}
\end{aligned}
$$

where $\xi=i \rho=e \sqrt{\vec{B}^{2}-\vec{E}^{2}}$. Note that $\xi$ can not be real in $D=2$ because $\vec{B}=0$. Thereby the potential (2.5) becomes [3, 4, 5, 6, 11]

$$
V(\sigma)=\frac{1}{2 \lambda} \sigma^{2}+\frac{1}{2} \operatorname{tr}[1] \int_{0}^{\infty} \frac{d s}{s} \frac{1}{(4 \pi s)^{D / 2}} e^{-s \sigma^{2}} s \xi \operatorname{coth}(s \xi) .
$$


Note that the external field dependence $s \xi \operatorname{coth} s \xi$, the loop momentum contribution $(4 \pi s)^{-D / 2}$ and the mass term $e^{-s \sigma^{2}}$ are decoupled in this expression.

Our proposal for introducing temperature and chemical potential is the replacement of the (normalization) factor $(4 \pi s)^{-D / 2}$ with a thermodynamic normalization. To see this, let us consider an alternative derivation of the second term on RHS of (2.9). It is the same as the one-loop effective action of a spinor loop (with mass $\sigma$ ) coupled to a background gauge (constant field strength) $A_{\mu}=\frac{1}{2} x^{\nu} F_{\nu \mu}$. In the worldline formalism, the effective action is organized in a form of closed path integral of one-dimensional Euclidean action 22, 23

$$
\begin{aligned}
\Gamma[A]= & \ln \operatorname{Det}\left(i \gamma^{\mu} D_{\mu}-\sigma\right) \\
= & \frac{1}{2} \int_{0}^{\infty} \frac{d s}{s} e^{-\sigma^{2} s} \oint \mathcal{D} x \mathcal{D} \psi \\
& \times \exp \left[-\int_{0}^{s} d \tau\left(\frac{1}{4} \dot{x}^{2}+\frac{1}{2} \psi \dot{\psi}+\frac{1}{2} i e x F \dot{x}-i e \psi F \psi\right)\right],
\end{aligned}
$$

where $x=x^{\mu}(\tau)$ and $\psi=\psi^{\mu}(\tau)$ are the periodic and anti-periodic world-line (real) fields which are defined on a closed loop parametrized by $\tau(0 \leq \tau \leq s)$, and $\dot{x}$ means $\partial_{\tau} x(\tau)$ etc. The closed path integral amounts to the super determinant

$$
\operatorname{SDet}^{-1 / 2}\left(\begin{array}{ll}
-\frac{1}{4} \partial_{\tau}^{2}+\frac{1}{2} i e F \partial_{\tau} & 0 \\
0 & \frac{1}{2} \partial_{\tau}-i e F
\end{array}\right)=\mathcal{N}\left[\frac{\operatorname{Det}\left(1-2 i e F \partial_{\tau}^{-1}\right)_{P}}{\operatorname{Det}\left(1-2 i e F \partial_{\tau}^{-1}\right)_{A}}\right]^{-1 / 2}
$$

where $\mathcal{N}$ is defined by the free part integral [22],

$$
\mathcal{N}=\oint \mathcal{D} x \mathcal{D} \psi \exp \left[-\int_{0}^{s}\left(\frac{1}{4} \dot{x}^{2}+\frac{1}{2} \psi \dot{\psi}\right) d \tau\right]
$$

and its explicit value is given by the formulae

$$
\begin{gathered}
\oint \mathcal{D} x \exp \left[-\int_{0}^{s} \frac{1}{4} \dot{x}^{2} d \tau\right]=\int \frac{d^{D} k}{(2 \pi)^{D}} e^{-s k^{2}}=(4 \pi s)^{-D / 2} \\
\oint \mathcal{D} \psi \exp \left[-\int_{0}^{s} \frac{1}{2} \psi \dot{\psi} d \tau\right]=-\operatorname{tr}[1]
\end{gathered}
$$

Thus the effective action yields

$$
\Gamma[A]=-\frac{1}{2} \operatorname{tr}[1] \int \frac{d s}{s}(4 \pi s)^{-D / 2} e^{-\sigma^{2} s}\left[\frac{\operatorname{Det}\left(1-2 i e F \partial_{\tau}^{-1}\right)_{A}}{\operatorname{Det}\left(1-2 i e F \partial_{\tau}^{-1}\right)_{P}}\right]^{1 / 2} .
$$


The determinants can be evaluated on the Fourier bases $\{\exp [2 \pi i k \tau / s] \mid k \in \mathbf{Z}, k \neq 0\}$ for the periodic and $\left\{\exp \left[2 \pi i\left(k+\frac{1}{2}\right) \tau / s\right] \mid k \in \mathbf{Z}\right\}$ for the anti-periodic functions [23]. Note that the zero mode in the periodic basis is excluded. We have

$$
\operatorname{Det}\left(1-2 i e F \partial_{\tau}^{-1}\right)_{P}=\operatorname{Det}^{\prime} \prod_{k=-\infty, \neq 0}^{\infty}\left(1-2 i e F \frac{s}{2 \pi i k}\right)=\operatorname{Det}^{\prime} \frac{\sin (e F s)}{e F s},
$$

and similarly

$$
\operatorname{Det}\left(1-2 i e F \partial_{\tau}^{-1}\right)_{A}=\operatorname{Det}^{\prime} \prod_{k=-\infty}^{\infty}\left(1-2 i e F \frac{s}{2 \pi i(k+1 / 2)}\right)=\operatorname{Det}^{\prime} \cos (e F s),
$$

where Det' concerns the determinant on a Lorentz matrix. We then arrive at the expression 23

$$
\Gamma[A]=-\frac{1}{2} \operatorname{tr}[1] \int \frac{d s}{s}(4 \pi s)^{-D / 2} e^{-\sigma^{2} s} \operatorname{Det}^{\prime \frac{1}{2}}[e F s \cot (e F s)],
$$

and this coincides with the second term of (2.9) with the identification of (2.6).

Now let us proceed to the thermodynamic case. We have to replace $k_{0}$-integral according to the Matsubara formalism

$$
k_{0} \rightarrow \frac{\pi}{\beta}(2 n+1)-i \mu, \quad \int \frac{d k_{0}}{2 \pi} \rightarrow \frac{1}{\beta} \sum_{n} .
$$

Namely, we change the path-integral normalization for $x_{0}$ through discretizing $k_{0}$-integral in $(2.13)$

$$
\begin{aligned}
\oint \mathcal{D} x_{0} \exp \left[-\int_{0}^{s} \frac{1}{4} \dot{x}_{0}^{2}\right] & \rightarrow \frac{1}{\beta} \sum_{n=-\infty}^{\infty} \exp \left[-s\left\{\frac{\pi}{\beta}(2 n+1)-i \mu\right\}^{2}\right] \\
& =\frac{1}{\beta} e^{s \mu^{2}} \Theta_{2}\left(s \frac{2 \mu}{\beta}, i s \frac{4 \pi}{\beta^{2}}\right),
\end{aligned}
$$

and (2.13) is then replaced by

$$
\oint_{\beta, \mu} \mathcal{D} x \exp \left[-\int_{0}^{s} \frac{1}{4} \dot{x}^{2}\right]=(4 \pi s)^{-\frac{D-1}{2}} \frac{1}{\beta} e^{s \mu^{2}} \Theta_{2}\left(s \frac{2 \mu}{\beta}, i s \frac{4 \pi}{\beta^{2}}\right) .
$$

Since the zero temperature limit $\beta \rightarrow \infty$ of RHS of $(2.20)$ is

$$
\lim _{\beta \rightarrow \infty} \frac{1}{\beta} e^{s \mu^{2}} \Theta_{2}\left(s \frac{2 \mu}{\beta}, i s \frac{4 \pi}{\beta^{2}}\right)=\frac{1}{\sqrt{4 \pi s}},
$$

(2.13) is reproduced from (2.21). Equivalently, this can be verified by decoupling the zero temperature normalization part $(4 \pi s)^{-D / 2}$,

$$
\begin{aligned}
\oint_{\beta, \mu} \mathcal{D} x \exp \left[-\int_{0}^{s} \frac{1}{4} \dot{x}^{2}\right] & =\oint \mathcal{D} x \exp \left[-\int_{0}^{s} \frac{1}{4} \dot{x}^{2}\right] \\
& +2(4 \pi s)^{\frac{-D}{2}} \sum_{n=1}^{\infty}(-1)^{n} e^{-n^{2} \beta^{2} / 4 s} \cosh (n \beta \mu),
\end{aligned}
$$


where we have applied the transformation

$$
\begin{aligned}
e^{s \mu^{2}} \frac{\sqrt{4 \pi s}}{\beta} \Theta_{2}\left(s \frac{2 \mu}{\beta}, i s \frac{4 \pi}{\beta^{2}}\right) & =\Theta_{4}\left(i \frac{\beta \mu}{2 \pi}, i \frac{\beta^{2}}{4 \pi s}\right) \\
& =1+2 \sum_{n=1}^{\infty}(-1)^{n} e^{-n^{2} \beta^{2} / 4 s} \cosh (n \beta \mu) .
\end{aligned}
$$

Obviously, the second term in (2.23) vanishes in $\beta \rightarrow \infty$, and is (2.13) recovered.

Let us remember that the thermal parameter $\beta^{-1}$ has nothing to do with the loop length $s$ which is the Schwinger proper time of the spinor loop [26]. All integrations on internal momenta $\left(k_{i}, k_{0}\right)$ are now done, and thus the operator determinants do not change any more, since $\partial_{\tau}$ acts on the proper time coordinate and $F$ is a constant matrix. We therefore conclude

$$
\Gamma[A]=-\frac{1}{2} \operatorname{tr}[1] \int \frac{d s}{s} \frac{\Theta_{2}\left(s \frac{2 \mu}{\beta}, i s \frac{4 \pi}{\beta^{2}}\right)}{(4 \pi s)^{(D-1) / 2} \beta} e^{s\left(\mu^{2}-\sigma^{2}\right)} s \xi \operatorname{coth}(s \xi) .
$$

Replacing the logarithmic part of (2.3) by this quantity, we get

$$
V_{\beta, \mu}(\sigma ; \xi)=\frac{1}{2 \lambda} \sigma^{2}+\frac{\operatorname{tr}[1]}{2 \beta} \int_{0}^{\infty} \frac{d s}{s} \frac{\Theta_{2}\left(s \frac{2 \mu}{\beta}, i s \frac{4 \pi}{\beta^{2}}\right)}{(4 \pi s)^{(D-1) / 2}} s \xi \operatorname{coth}(s \xi) e^{-s\left(\sigma^{2}-\mu^{2}\right)}
$$

When $D=3$, this thermodynamic potential coincides with the equation (B6) of Ref. 111. Also, when $\xi=0, V_{\beta, \mu}(\sigma ; \xi=0)$ coincides with the $D$-dimensional thermodynamic potential discussed in [19. When $T=\mu=0, V_{\beta, \mu}(\sigma ; \xi)$ is reduced to (2.9) because of (2.22). Finally, subtracting the zero point value of the potential, we obtain the announced result (2.4)

$$
V(\sigma ; \xi, \beta, \mu)=V_{\beta, \mu}(\sigma ; \xi)-V_{\beta, \mu}(0 ; \xi)
$$

\subsection{The critical equations}

In the following, we list the critical equations that are entire bases of our numerical analyses of chiral phase structures. We should remember that the original effective potential (2.3) contains the ultra-violet divergence and also has an imaginary part for pure imaginary $\xi$. When the imaginary part disappears, we shall impose the following renormalization condition (on the real part)

$$
\left.\lim _{T, \mu \rightarrow 0} \frac{\partial^{2}}{\partial \sigma^{2}} \operatorname{Re} V(\sigma ; \xi, T, \mu)\right|_{\sigma=1}=\frac{1}{\lambda_{R}}
$$


and we can thus define the renormalized coupling constant $\lambda_{R}$ in the following way

$$
\frac{1}{\lambda}-\frac{1}{\lambda_{R}}=\operatorname{tr}[1] \operatorname{Re} \int_{0}^{\infty} \frac{d s}{(4 \pi s)^{D / 2}} e^{-s}(1-2 s) s \xi \operatorname{coth}(s \xi) .
$$

The real part of renormalized effective potential is then expressed in terms of $\lambda_{R}$ as

$$
\begin{aligned}
\operatorname{Re} V_{R}(\sigma ; \xi, T, \mu) & =\frac{1}{2 \lambda_{R}} \sigma^{2}+\frac{1}{2} \operatorname{tr}[1] \operatorname{Re} \int_{0}^{\infty} \frac{d s}{(4 \pi s)^{D / 2}} s \xi \operatorname{coth}(s \xi) \\
& \times\left[\frac{1}{s}\left(e^{-s \sigma^{2}}-1\right) \frac{\sqrt{4 \pi s}}{\beta} e^{s \mu^{2}} \Theta_{2}\left(s \frac{2 \mu}{\beta}, i s \frac{4 \pi}{\beta^{2}}\right)+\sigma^{2} e^{-s}(1-2 s)\right] .
\end{aligned}
$$

The gap equation to determine the dynamical mass, i.e., the lowest energy configuration of $\sigma$, is then

$$
\begin{aligned}
0= & \frac{1}{\lambda_{R}}+\operatorname{tr}[1] \operatorname{Re} \int_{0}^{\infty} \frac{d s}{(4 \pi s)^{D / 2}} s \xi \operatorname{coth}(s \xi) \\
& \times\left[-e^{-s\left(\sigma^{2}-\mu^{2}\right)} \frac{\sqrt{4 \pi s}}{\beta} \Theta_{2}\left(s \frac{2 \mu}{\beta}, i s \frac{4 \pi}{\beta^{2}}\right)+e^{-s}(1-2 s)\right],
\end{aligned}
$$

where we have eliminated the trivial solution $\sigma=0$.

Now let us derive the equations to determine phase diagrams in the $\xi-T-\mu$ parameter space. The critical surface of first order is governed by the simultaneous equations

$$
\begin{gathered}
\operatorname{Re} V_{R}(\sigma=m, \xi, \beta, \mu)=0, \\
\left.\frac{\partial}{\partial \sigma} \operatorname{Re} V_{R}(\sigma, \xi, \beta, \mu)\right|_{\sigma=m}=0,
\end{gathered}
$$

where $m$ denotes a non-trivial solution of (2.31). Combining these equations, we obtain

$$
\begin{aligned}
0= & \operatorname{Re} \int_{0}^{\infty} \frac{d s}{(4 \pi s)^{D / 2}} s \xi \operatorname{coth}(s \xi) \cdot \frac{\sqrt{4 \pi s}}{\beta} e^{s \mu^{2}} \Theta_{2}\left(s \frac{2 \mu}{\beta}, i s \frac{4 \pi}{\beta^{2}}\right) \\
& \times\left[m^{2} e^{-s m^{2}}+\frac{1}{s}\left(e^{-s m^{2}}-1\right)\right] .
\end{aligned}
$$

One can decouple RHS of this equation into $V(m ; \xi)$ and a pure thermal part using (2.24), however we stop writing further details, which will be discussed in sect.3.1. The necessary condition to determine the critical surface of second order is generally expressed by

$$
\lim _{\sigma \rightarrow 0} \frac{\partial}{\partial \sigma^{2}} \operatorname{Re} V_{R}(\sigma, \xi, \beta, \mu)=0,
$$

and this reads

$$
\begin{gathered}
0=\operatorname{Re} \int_{0}^{\infty} \frac{d s}{(4 \pi s)^{D / 2}}[ \\
s \xi \operatorname{coth}(s \xi)\left\{-e^{s \mu^{2}} \frac{\sqrt{4 \pi s}}{\beta} \Theta_{2}\left(s \frac{2 \mu}{\beta}, i s \frac{4 \pi}{\beta^{2}}\right)\right. \\
\left.\left.+e^{-s}(1-2 s)\right\}+2 s e^{-s}\right] .
\end{gathered}
$$


Here, we have adopted the following value of the renormalized coupling constant

$$
\frac{1}{\lambda_{R}}=\operatorname{tr}[1] \int_{0}^{\infty} \frac{d s}{(4 \pi s)^{D / 2}} 2 s e^{-s}
$$

which makes the chiral symmetry broken at $T=\mu=\xi=0$ with generating the dynamical mass $\sigma=1$. Note that a solution of (2.36) does not always represent a true second order transition point if a first order transition occurs. We have to cut an irrelevant piece away from the true critical surface solving the equation of third order critical line. The third order critical line, which yields the boundary between the first and second order critical surfaces, is determined by the simultaneous equations (2.35) and

$$
\lim _{\sigma \rightarrow 0}\left(\frac{\partial}{\partial \sigma^{2}}\right)^{2} \operatorname{Re} V_{R}(\sigma, \xi, \beta, \mu)=0 .
$$

The latter equation leads to

$$
0=\operatorname{Re} \int_{0}^{\infty} \frac{s d s}{(4 \pi s)^{D / 2}} s \xi \operatorname{coth}(s \xi) e^{s \mu^{2}} \frac{\sqrt{4 \pi s}}{\beta} \Theta_{2}\left(s \frac{2 \mu}{\beta}, i s \frac{4 \pi}{\beta^{2}}\right) .
$$

One can perform any combination of limits $\xi, T$ and/or $\mu \rightarrow 0$ in the 2'nd and 3'rd order critical equations (2.36) and (2.39). For instance, taking the limit $\xi \rightarrow 0$ in each equation, we obtain the following analytic equations respectively [17, 19 (see Appendix A)

$$
\beta^{D-2} \Gamma\left(1-\frac{D}{2}\right)=\frac{2}{\sqrt{\pi}}(2 \pi)^{D-2} \Gamma\left(\frac{3-D}{2}\right) \operatorname{Re} \zeta\left(3-D, \frac{1}{2}+i \frac{\beta \mu}{2 \pi}\right),
$$

and

$$
\operatorname{Re} \zeta\left(5-D, \frac{1}{2}+i \frac{\beta \mu}{2 \pi}\right)=0
$$

where $\zeta$ is the generalized zeta function. It is shown in [19] that these equations reproduce the critical equations derived in [18]. The limit $\beta \rightarrow \infty$ can also be taken by applying (2.22) to (2.36) and (2.39), and their limits are valid unless any 1'st order phase transition takes place at $T=0$. In contrast with these cases, the effective potential on the $\xi$ - $\mu$ plane (and hence the 1'st order critical equation there) can not be obtained for $\mu \neq 0$ by naively applying (2.22). In order to reproduce a precise limit, we have to collect all contributions from under the Fermi surface at $T=0$. This can be achieved by an insertion 
of the step function $\theta(\mu-k)$ in a loop momentum integration [20] at a very beginning of computation. This prescription is, however, unclear in our present formulation, since we already integrated the loop momenta. We observe a method how to deal with this problem in the next section.

\section{The magnetic dominant cases}

In this section, we discuss chiral phase structures in the magnetic dominant cases for finite $T$ and $\mu$. We formally include $D=2$ case also, because it will be useful when $\xi$ is rotated to $i \rho$ and because it is convenient to see a $D$-dependence of magnetic catalyst in the region $2<D<4$. Since $\xi$ is real, the effective potential does not possess an imaginary part, i.e.,

$$
\operatorname{Re} V_{R}(\sigma ; \xi, \beta, \mu)=V_{R}(\sigma ; \xi, \beta, \mu)
$$

and there is no more intricacy of critical equation analysis (up to the $T \rightarrow 0$ limit). In [11], the $D=3$ case $(\mu=0, T \neq 0)$ with a constant magnetic field was analyzed, and the magnetic field turns out to play a role of strong catalyst of chiral symmetry breaking. We shall observe this feature in the $\xi-T-\mu$ space for various values of $D$. Each critical surface is determined by numerically analyzing the 1'st, 2'nd and 3'rd order critical equations presented in the previous section. As to the zero temperature limit, expected from [8], we shall show that first order phase transitions exist on the $\xi$ - $\mu$ plane at large values of $\mu$.

\subsection{The effective potential on $\xi-\mu$ plane}

First we study the zero temperature limit for finite $\xi$ and $\mu$. As mentioned at the end of sect.2, the special care is necessary to analyze the effective potential (not gap equation)

on the $\xi$ - $\mu$ plane. In addition, we have to remember that $\xi^{-1}$ is relevant to the magnetic length $e B$ (or $\xi$ the discrete momentum unit), which is known as the radius of cyclotron motion of an electron. This assures the magnetic lattice structure, although it can not be seen in the theta function representations of the effective potential (2.30) etc.

The effective potential (2.27) with the renormalization (2.29) can be written as the sum of the zero-temperature potential (2.2) and the pure thermodynamic remainder $\tilde{V}$ by 
means of the formula (2.24):

$$
V(\sigma ; \xi, \beta, \mu)=V(\sigma ; \xi)+\tilde{V}(\sigma ; \xi, \beta, \mu)
$$

or (q.v. (2.2) and (2.27))

$$
V_{\beta, \mu}(\sigma ; \xi)=V(\sigma)+\tilde{V}_{\beta, \mu}(\sigma ; \xi)
$$

where

$$
\begin{gathered}
V(\sigma)=\frac{1}{2 \lambda_{R}} \sigma^{2}+\frac{\operatorname{tr}[1]}{2} \int_{0}^{\infty} \frac{d s}{(4 \pi s)^{D / 2}} s \xi \operatorname{coth}(s \xi)\left[\frac{1}{s} e^{-s \sigma^{2}}+\sigma^{2} e^{-s}(1-2 s)\right], \\
\tilde{V}_{\beta, \mu}(\sigma ; \xi)=\operatorname{tr}[1] \int_{0}^{\infty} \frac{d s}{(4 \pi s)^{D / 2}} \xi \operatorname{coth}(s \xi) \cdot e^{-s \sigma^{2}} \sum_{n=1}^{\infty}(-1)^{n} e^{-n^{2} \beta^{2} / 4 s} \cosh (n \beta \mu) .
\end{gathered}
$$

Using the following formulae

$$
\begin{gathered}
\operatorname{coth}(s \xi)=1+2 \sum_{l=1}^{\infty} e^{-2 l s \xi} \\
\int_{0}^{\infty} d s s^{\nu-1} \exp \left[-\frac{\beta}{s}-\gamma s\right]=2\left(\frac{\beta}{\gamma}\right)^{\frac{\nu}{2}} K_{\nu}(2 \sqrt{\beta \gamma}),
\end{gathered}
$$

we can perform the $s$-integrations in (3.4) and (3.5) just similarly to the $D=3$ case 11$]$. The results are

$$
\begin{aligned}
V(\sigma)=\frac{\sigma^{2}}{2 \lambda_{R}}+ & \frac{\operatorname{tr}[1] \xi}{2(4 \pi)^{D / 2}}\left[2(2 \xi)^{\frac{D}{2}-1} \zeta\left(1-\frac{D}{2}, 1+\frac{\sigma^{2}}{2 \xi}\right) \Gamma\left(1-\frac{D}{2}\right)+\sigma^{D-2} \Gamma\left(1-\frac{D}{2}\right)\right. \\
& +2 \sigma^{2}(2 \xi)^{\frac{D}{2}-2} \zeta\left(2-\frac{D}{2}, 1+\frac{1}{2 \xi}\right) \Gamma\left(2-\frac{D}{2}\right)+\sigma^{2} \Gamma\left(2-\frac{D}{2}\right) \\
& \left.-4 \sigma^{2}(2 \xi)^{\frac{D}{2}-3} \zeta\left(3-\frac{D}{2}, 1+\frac{1}{2 \xi}\right) \Gamma\left(3-\frac{D}{2}\right)-2 \sigma^{2} \Gamma\left(3-\frac{D}{2}\right)\right]
\end{aligned}
$$

and

$$
\tilde{V}_{\beta, \mu}(\sigma ; \xi)=\frac{\operatorname{tr}[1] \xi}{(4 \pi)^{D / 2}}\left[\frac{1}{2} \mathcal{O}_{\beta}(\sigma)+\sum_{l=1}^{\infty} \mathcal{O}_{\beta}\left(\sqrt{\sigma^{2}+2 l \xi}\right)\right],
$$

where we have introduced the compact notation

$$
\begin{aligned}
\mathcal{O}_{\beta}(\sigma) & \equiv 2 \int_{0}^{\infty} d s s^{-D / 2} \sum_{n=1}^{\infty}(-1)^{n} \cosh (n \beta \mu) \exp \left[-\left(s \sigma^{2}+\frac{n^{2} \beta^{2}}{4 s}\right)\right] \\
& =4 \sum_{n=1}^{\infty}(-1)^{n} \cosh (n \beta \mu)\left(\frac{\beta n}{2 \sigma}\right)^{1-D / 2} K_{D / 2-1}(n \beta \sigma) .
\end{aligned}
$$


Although we have performed the integrations w.r.t. the proper time $s$, it is rather convenient to consider an integral representation in order to observe the low temperature limit of $\mathcal{O}_{\beta}(\sigma)$. Using the formula

$$
K_{\nu}(z)=\frac{\sqrt{\pi}\left(\frac{z}{2}\right)^{\nu}}{\Gamma\left(\nu+\frac{1}{2}\right)} \int_{1}^{\infty} e^{-z t}\left(t^{2}-1\right)^{\nu-\frac{1}{2}} d t, \quad \operatorname{Re} \nu>-\frac{1}{2}, \quad \operatorname{Re} z>0
$$

we get the following integral representation

$$
\mathcal{O}_{\beta}(\sigma)=\frac{4 \sqrt{\pi}}{\Gamma\left(\frac{D-1}{2}\right)} \sigma^{D-2} \sum_{n=1}^{\infty}(-)^{n} \cosh (n \beta \mu) \int_{1}^{\infty} e^{-n \beta \sigma t}\left(t^{2}-1\right)^{\frac{D-3}{2}} d t
$$

Performing the summation over $n$ and changing the integration variable $t=1+t^{\prime} / \sigma$, we obtain

$$
\mathcal{O}_{\beta}(\sigma)=\frac{-2 \sqrt{\pi}}{\Gamma\left(\frac{D-1}{2}\right)}\left[\int_{0}^{\infty} \frac{\left(t^{2}+2 \sigma t\right)^{\frac{D-3}{2}}}{1+e^{\beta(t+\sigma+\mu)}} d t \quad+(\mu \rightarrow \mu)\right] .
$$

$\mathcal{O}_{\beta}(0)$ is given by (a derivation is in Appendix A)

$$
\mathcal{O}_{\beta}(0)=\frac{2}{\sqrt{\pi}}\left(\frac{2 \pi}{\beta}\right)^{D-2} \Gamma\left(\frac{3-D}{2}\right) \operatorname{Re} \zeta\left(3-D, \frac{1}{2}+i \frac{\beta \mu}{2 \pi}\right)
$$

Here, we stress that (3.9) is a discrete version of the $\xi=0$ potential obtained in 19

$$
\tilde{V}(\sigma ; 0, \beta, \mu)=-\frac{\operatorname{tr}[1] 2 \sqrt{\pi}}{(4 \pi)^{D / 2} \Gamma\left(\frac{D-1}{2}\right) \beta} \int_{0}^{\infty} d k k^{D-2} \ln \frac{\left(1+e^{-\beta\left(\mu+\sqrt{\sigma^{2}+k^{2}}\right)}\right)\left(1+e^{-\beta\left(\sqrt{\sigma^{2}+k^{2}}-\mu\right)}\right)}{\left(1+e^{-\beta(\mu+k)}\right)\left(1+e^{-\beta(k-\mu)}\right)} .
$$

This can be easily understood in the case of $D=3$, since $\mathcal{O}_{\beta}(\sigma)$ takes the following simple form

$$
\mathcal{O}_{\beta}(\sigma)=-2 \frac{\sqrt{\pi}}{\beta} \ln \left[\left(1+e^{-\beta(\sigma+\mu)}\right)\left(1+e^{-\beta(\sigma-\mu)}\right)\right],
$$

and hence we have (see also [11, 24])

$$
\begin{aligned}
\tilde{V}(\sigma ; \xi, \beta, \mu) & =\tilde{V}_{\beta, \mu}(\sigma ; \xi)-\tilde{V}_{\beta, \mu}(0 ; \xi) \\
& =-\frac{\operatorname{tr}[1] \xi 2 \sqrt{\pi}}{(4 \pi)^{3 / 2} \beta} \frac{1}{2} \ln \frac{\left(1+e^{\beta(\mu-\sigma)}\right)\left(1+e^{-\beta(\mu+\sigma)}\right)}{\left(1+e^{\beta \mu}\right)\left(1+e^{-\beta \mu}\right)} \\
& \left.+\sum_{l=1}^{\infty} \ln \frac{\left(1+e^{\beta\left(\mu-\sqrt{\sigma^{2}+2 l \xi}\right)}\right)\left(1+e^{-\beta\left(\mu+\sqrt{\sigma^{2}+2 l \xi}\right)}\right)}{\left(1+e^{\beta(\mu-\sqrt{2 l \xi})}\right)\left(1+e^{-\beta(\mu+\sqrt{2 l \xi})}\right)}\right] .
\end{aligned}
$$

This is exactly a discrete summation form of (3.15), and the correspondence to (3.15) is understood by the following translation rule from discrete spectrum to continuum one

$$
2 l \xi \quad \rightarrow \quad k^{2},
$$




$$
\begin{aligned}
\xi & \rightarrow k d k, \\
\xi \sum_{l=1}^{\infty} & \rightarrow \int_{0}^{\infty} k d k
\end{aligned}
$$

Remember, in the continuum expression, we have to (i) insert a step function $\theta(\mu-k)$ into the above momentum integration and have to (ii) keep $\mu<\sigma$ in order to observe a correct potential behavior in $\beta \rightarrow \infty$ with finite $\mu$; namely [19

$$
\lim _{\beta \rightarrow \infty} \tilde{V}(\sigma ; 0, \beta, \mu)=\frac{\operatorname{tr}[1] 2 \sqrt{\pi}}{(4 \pi)^{D / 2} \Gamma\left(\frac{D-1}{2}\right)} \int_{0}^{\mu} d k k^{D-2}(\mu-k) .
$$

Due to the correspondence (3.18), we may similarly assume an upper bound on the discrete momentum sum; $l<L \equiv\left[\mu^{2} / 2 \xi\right]_{G}$, where [ $]_{G}$ is the Gauss symbol,

$$
\lim _{\beta \rightarrow \infty} \tilde{V}(\sigma ; \xi, \beta, \mu)=\frac{\operatorname{tr}[1] \xi 2 \sqrt{\pi}}{(4 \pi)^{3 / 2}}\left[\frac{1}{2} \mu+\sum_{l=1}^{L}(\mu-\sqrt{2 l \xi})\right] .
$$

This potential is still clearly a discrete form of (3.19) for $D=3$ under the identification of (3.18).

Let us return to the generic $D$ case. The above cutting procedure is also valid for the generic $D$ case, because we naturally have a step function in

$$
\begin{aligned}
\mathcal{O}_{\infty}(\sigma) & \equiv \lim _{\beta \rightarrow \infty} \mathcal{O}_{\beta}(\sigma) \\
& =-\frac{2 \sqrt{\pi}}{\Gamma\left(\frac{D-1}{2}\right)} \theta(\mu-\sigma) \int_{0}^{\mu-\sigma}\left(t^{2}+2 \sigma t\right)^{\frac{D-3}{2}} d t \\
& =-\frac{2 \sqrt{\pi}}{\Gamma\left(\frac{D+1}{2}\right)}(2 \sigma)^{\frac{D-3}{2}}(\mu-\sigma)^{\frac{D-1}{2}} F\left(\frac{3-D}{2}, \frac{D-1}{2} ; \frac{D+1}{2} ; \frac{\sigma-\mu}{2 \sigma}\right) \theta(\mu-\sigma),
\end{aligned}
$$

where we have applied the integration formula (B.3). Owing to the presence of the step function $\theta(\mu-\sigma)$, the upper bound $L$ is naturally introduced here, and we figure out

$$
\lim _{\beta \rightarrow \infty} \tilde{V}(\sigma ; \xi, \beta, \mu)=-\frac{\operatorname{tr}[1] \xi}{(4 \pi)^{D / 2}}\left[\frac{1}{2} \mathcal{O}_{\infty}(0)+\sum_{l=1}^{L} \mathcal{O}_{\infty}(\sqrt{2 l \xi})\right] .
$$

This is the discrete form corresponding to (3.19). The explicit forms of $\mathcal{O}_{\infty}(\sigma)$ in each $D$ are

$$
\mathcal{O}_{\infty}(\sigma)=\frac{-2 \sqrt{\pi}}{\Gamma\left(\frac{D-1}{2}\right)} \theta(\mu-\sigma) \times \begin{cases}\operatorname{arccosh}(\mu / \sigma) & \text { for } D=2 \\ \mu-\sigma & \text { for } D=3 \\ \frac{1}{2} \mu \sqrt{\mu^{2}-\sigma^{2}}-\frac{1}{2} \sigma^{2} \operatorname{arccosh}(\mu / \sigma) & \text { for } D=4,\end{cases}
$$


and

$$
\mathcal{O}_{\infty}(0)=-\frac{2 \sqrt{\pi}}{\Gamma\left(\frac{D-1}{2}\right)} \frac{\mu^{D-2}}{D-2} .
$$

The $\mathcal{O}_{\infty}(0)$ is singular when $D=2$, however it vanishes under the 'physical' limit $\xi \rightarrow 0$. In Appendix B, we show how the discrete form (3.22) reproduces the continuum one (3.19) in the limit $\xi \rightarrow 0$ via $(3.18)$.

Now let us examine the phase structure on the $\xi-\mu$ plane. It is enough to analyze the 1 'st order critical equation defined by $(2.32)$ and (2.33). The reason will become clear at the bottom of this paragraph. Since the pure thermal part (3.22) does not depend on $\sigma$ any more, the gap equation is the same as the one for the zero temperature potential $V(\sigma ; \xi)$

$$
0=\frac{1}{\lambda_{R}}+\operatorname{tr}[1] \int_{0}^{\infty} \frac{d s}{(4 \pi s)^{D / 2}} s \xi \operatorname{coth}(s \xi)\left[e^{-s}(1-2 s)-e^{-s m^{2}}\right] .
$$

(Of course, this can be derived from (2.31) with the use of (2.22).) Eliminating the renormalized coupling from (3.4) with using (3.25), and applying the following formula f

$$
\int_{0}^{\infty} d s s^{\mu-1} e^{-\beta s} \operatorname{coth} s=\Gamma(\mu)\left[2^{1-\mu} \zeta\left(\mu, 1+\frac{\beta}{2}\right)+\beta^{-\mu}\right], \quad \operatorname{Re} \mu>1
$$

$\left.V(\sigma ; \xi)\right|_{\sigma=m}$ turns out to be

$$
\begin{aligned}
V(m ; \xi) & =\frac{\operatorname{tr}[1] 2^{D / 2} \xi^{\frac{D}{2}-1}}{2(4 \pi)^{D / 2}} \Gamma\left(1-\frac{D}{2}\right)\left[\frac{1}{2} m^{2}\left(1-\frac{D}{2}\right) \zeta\left(2-\frac{D}{2}, 1+\frac{m^{2}}{2 \xi}\right)\right. \\
& \left.+\xi \zeta\left(1-\frac{D}{2}, 1+\frac{m^{2}}{2 \xi}\right)-\xi \zeta\left(1-\frac{D}{2}\right)+\frac{m^{2}}{4}\left(2-\frac{D}{2}\right)\left(\frac{2 \xi}{m^{2}}\right)^{2-D / 2}\right] .
\end{aligned}
$$

Combining this and (3.22), we obtain the 1'st order critical equation on the $\xi$ - $\mu$ plane

$$
\begin{aligned}
0 & =\frac{-2}{\Gamma\left(1-\frac{D}{2}\right)}(2 \xi)^{2-\frac{D}{2}}\left[\frac{1}{2} \mathcal{O}_{\infty}(0)+\sum_{l=1}^{L} \mathcal{O}_{\infty}(\sqrt{2 l \xi})\right] \\
& +m^{2}(2-D) \zeta\left(2-D, 1+\frac{m^{2}}{2 \xi}\right)+\frac{4-D}{2}(2 \xi)^{2-\frac{D}{2}} m^{D-2} \\
& +4 \xi \zeta\left(1-\frac{D}{2}, 1+\frac{m^{2}}{2 \xi}\right)-4 \xi \zeta\left(1-\frac{D}{2}\right)
\end{aligned}
$$

where $m$ should be determined from (3.25). (For example, we can take $m=1$ as a solution if we choose, instead of (2.37),

$$
\frac{1}{\lambda_{R}}=\operatorname{tr}[1] \int_{0}^{\infty} \frac{d s}{(4 \pi s)^{D / 2}} s \xi \operatorname{coth}(s \xi) \cdot 2 s e^{-s} .
$$

\footnotetext{
4 This formula is derived from the expansion $(3.6$ ) and the definition of zeta function.
} 
This coupling choice makes our critical equations much simpler, however it is not essential for the numerical analyses of phase structures discussed later). Under the choice (2.37), the mass 甲 is determined by

$$
\begin{aligned}
0= & 2-\xi(3-D)-\xi m^{D-4}-(2 \xi)^{\frac{D}{2}-1} \zeta\left(2-\frac{D}{2}, 1+\frac{m^{2}}{2 \xi}\right) \\
& +(2 \xi)^{\frac{D}{2}-1} \zeta\left(2-\frac{D}{2}, 1+\frac{1}{2 \xi}\right)-(2 \xi)^{\frac{D}{2}-2}(4-D) \zeta\left(3-\frac{D}{2}, 1+\frac{1}{2 \xi}\right) .
\end{aligned}
$$

The dynamical mass determined by (3.30) grows as the strength $\xi$ of the external magnetic field becoming large (see Fig.1), whose feature coincides with the one argued in [8]. Note that we can not take the limit $m \rightarrow 0$ without circumventing divergence for $D<4$ in this equation. This means there is no 2'nd order phase transition on the $\xi-\mu$ plane.

Substituting the solutions of (3.30) into (3.28), we find 1'st order transitions (see Fig.2) as the solution of (3.28). The equation (3.28) can be shown to coincide with the following equation [19] in the limit $\xi \rightarrow 0$ (The proof is in Appendix C),

$$
\left(\frac{\mu}{m}\right)^{D}=\frac{D-1}{2}\left(1-\frac{D}{2}\right) \mathrm{B}\left(1-\frac{D}{2}, \frac{D-1}{2}\right),
$$

which has the solutions $\mu=m / \sqrt{2}$ for $D=2$ and $\mu=m$ for $D=3$ [18, 20].

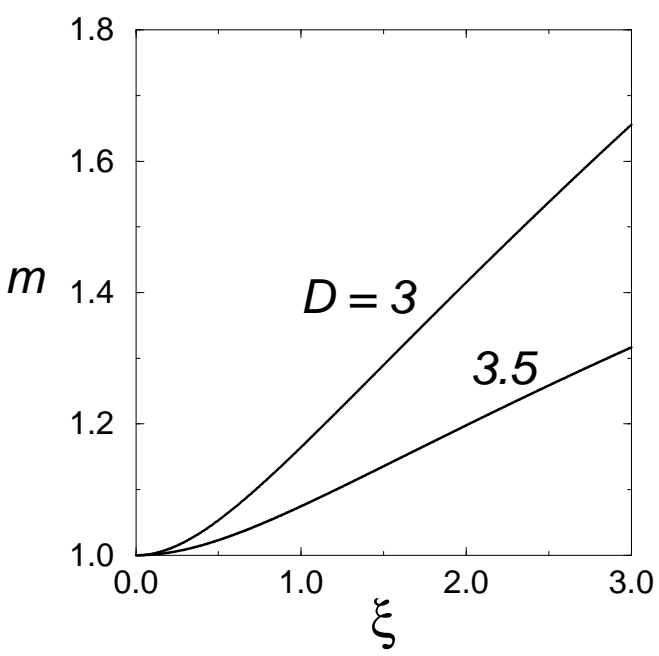

Fig. 1: The dynamical mass $m$ for magnetic dominant cases on $T=0$ plane.

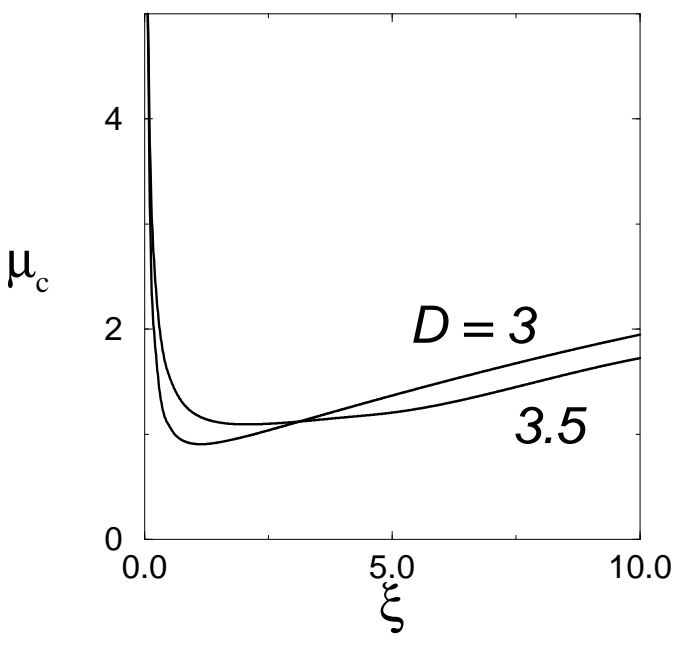

Fig. 2: The values of critical chemical potential $\mu_{c}$ on the $T=0$ plane as a function of the external magnetic field $\xi$.

\footnotetext{
${ }^{5}$ For example, a solution is $m=1.02107$ for $\xi=0.3$ in $D=3$.
} 


\subsection{The phase structures}

In Fig.3, we illustrate several critical curves which represent various sections of the second order critical surface in the $\xi-T$ - $\mu$ space for $D=3$. In this case, the point $(\xi, T, \mu)=$ $(0,0,1)$ is the unique tricritical point, since (2.41) has the solution $(\beta \mu)^{-1}=0$, which means $T=0$ (for $\mu \neq 0$ ). Fig.4 is the case of $D=4-\epsilon$, where we take $\epsilon=0.5$. No tricritical point appears in this case. We could not let $\epsilon$ to be much smaller than this value due to a singular situation of numerical computations. However we can easily read some signs of the magnetic catalyst and the disappearance of the tricritical point, if we extrapolate from the following behaviors of larger values $\epsilon=1.5$ and 2.0.

First, let us observe the $D=2.5(\epsilon=1.5)$ diagrams in Fig.5, where the tricritical point starts appearing around $0.75<\mu<0.76$. These tricritical points are determined from intersections between two curves solved from (2.36) and (2.39) like the way shown in Fig.6. Next, let us watch the $D=2$ diagrams (Fig.7). The tricritical line (points) and first order transitions are observed for $0.61<\mu$. The phase diagram is very similar to Fig.5, and these diagrams exhibit that strong $\xi$ makes phase broken (magnetic catalyst) even in these unphysical dimensions $2 \leq D<3$. Now we gather the tricritical points of each dimension in Fig.8. It clearly shows that the tricritical line moves toward a lower temperature region as $D$ increasing. And $D=3$ is the terminal case that the tricritical point appears. We therefore deduce that there is no tricritical point in $D=4-\epsilon$ for small $\epsilon$ whilst observing the magnetic catalyst in finite $T$ and $\mu$.

Let us summarize the phase structures. We have observed that the critical surfaces in $D=3$ and $4-\epsilon$ are of second order, and magnetic fields tend to make the chiral symmetry broken even at finite $T$ and $\mu$. The large values of $\mu$ tend to revive the chiral symmetry, and the critical $\mu$ grows as $\xi$ increasing (for all $T$ ). There are first order transitions not at finite $T$ but at zero temperature shown in Fig.2, where the $\xi=0$ axis is singular and discontinuous. 


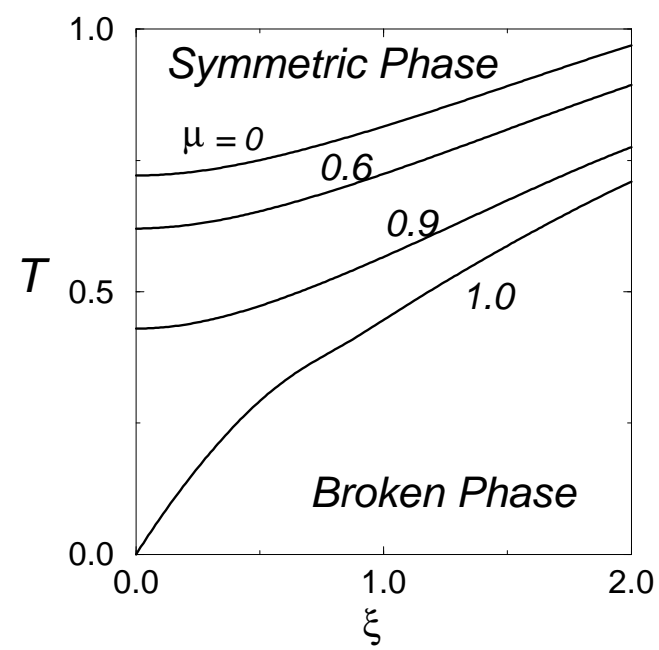

Fig. 3. The critical lines for magnetic dominant cases in $D=3$.

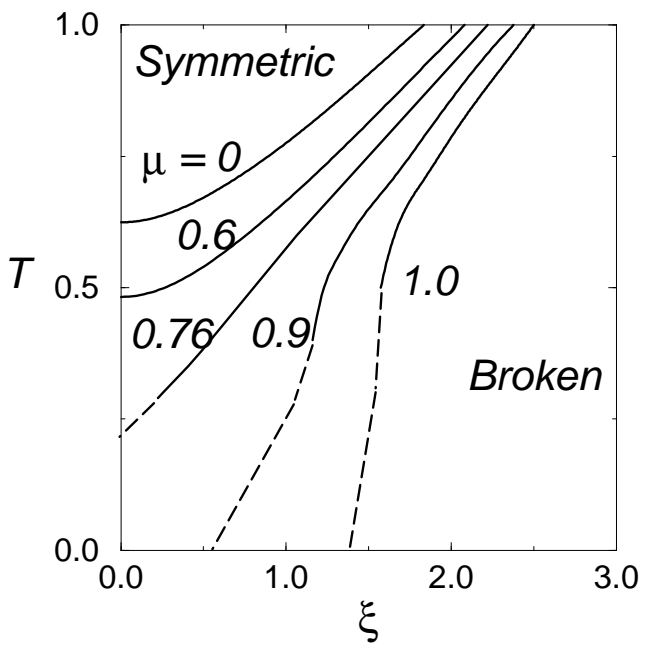

Fig. 5: The critical lines for magnetic dominant cases in $D=2.5$.

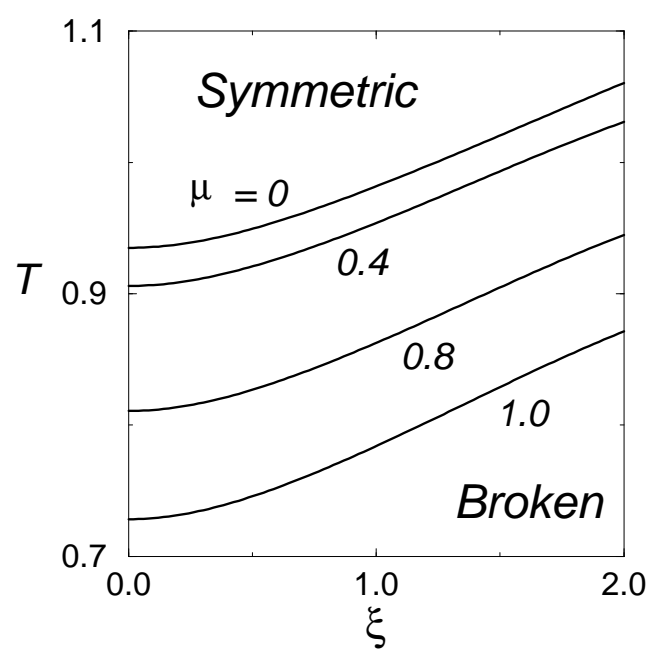

Fig. 4: The critical lines for magnetic dominant cases in $D=4-\epsilon$, where $\epsilon$ is chosen to be 0.5 .

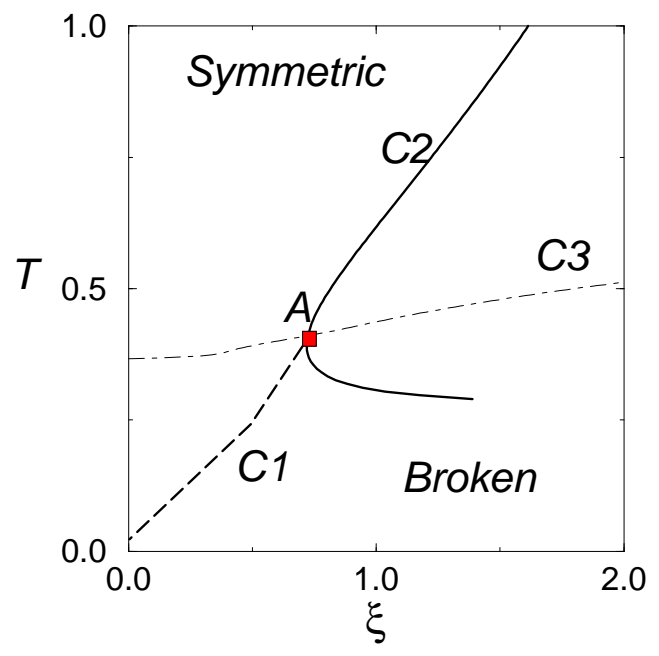

Fig. 6: How to determine the tricritical point $A$. This example is the case of $\mu=0.7, D=2$ (magnetic dominant). The $C 2$ and $C 3$ are the solutions of eqs. (2.36) and (2.39). The $C 1$ represents the solution of (2.34). 


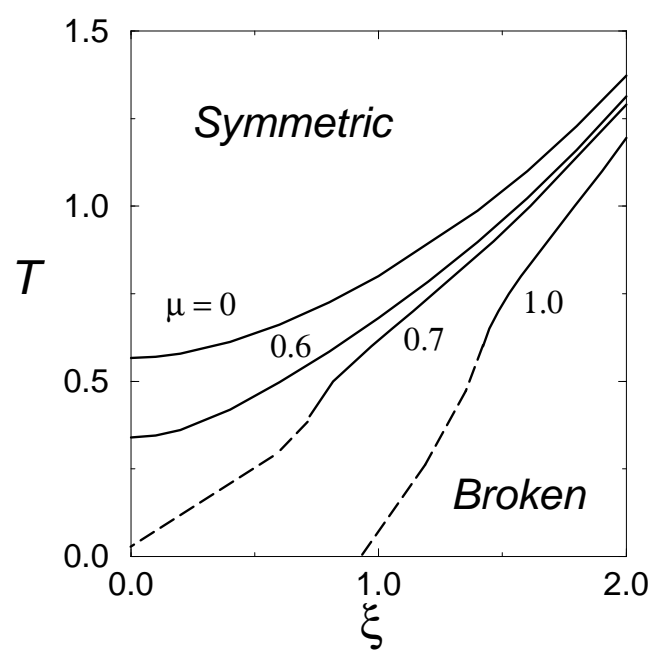

Fig. 7: The critical lines in $D=2$ for magnetic-like external field. The $T=0$ plane is singular.

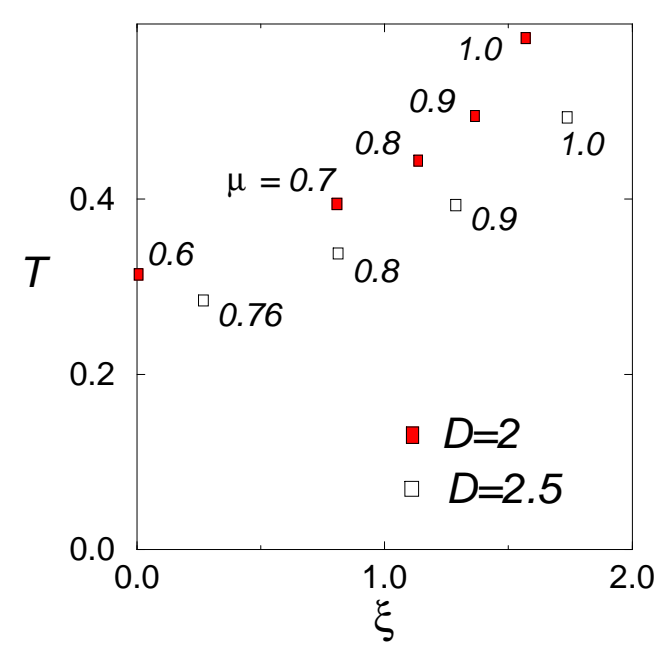

Fig. 8: The tricritical points projected on the $T-\xi$ plane for magnetic dominant cases.

\section{The electric dominant cases}

\subsection{The imaginary part of effective potential}

In contrast with the $B$-dominant cases, the effective potential (2.4) gives rise to an imaginary part due to the instability of fermion vacuum in the $E$-dominant cases [3, 25, 26]. In fact, the function $s \xi \operatorname{coth}(s \xi)$ in the integrand of (2.4) is now $s \rho \cot (s \rho)$ with changing $\xi=i \rho$, and the integrand then contains an infinite number of the singular points $s=$

$n \pi / \rho,(n=1,2, \cdots)$ accordingly. Then we must displace the contour of integration $(-\infty<$ $s<\infty)$ by $i \epsilon$ along the imaginary axis of the complex $s$-plane. The contribution of each singular point is taken into account by making use of the $i \epsilon$ rule (for example, 25 )

$$
\frac{1}{s+i \epsilon-\frac{n \pi}{\rho}}=\frac{\mathrm{P}}{s-\frac{n \pi}{\rho}}-i \pi \delta\left(s-\frac{n \pi}{\rho}\right),
$$

where $\mathrm{P}$ stands for the principal-value symbol. By virtue of this rule, the imaginary part 
of the effective potential consists of

$$
\begin{aligned}
\operatorname{Im} V_{\beta, \mu}(\sigma ; \rho)= & -\operatorname{tr}[1] \frac{\rho^{(D-1) / 2}}{2^{D} \pi^{D-2} \beta} \sum_{n=1}^{\infty} n^{\frac{1-D}{2}} e^{-n \pi\left(\sigma^{2}-\mu^{2}\right) / \rho} \Theta_{2}\left(\frac{2 n \pi \mu}{\rho \beta}, i \frac{4 n \pi^{2}}{\rho \beta^{2}}\right) \\
= & -\operatorname{tr}[1] \frac{\rho^{(D-1) / 2}}{2^{D-1} \pi^{D-2} \beta} \operatorname{Re} \sum_{m=0}^{\infty} \exp \left[-\frac{\pi}{\rho}\left\{\sigma^{2}+\left(\pi \frac{2 m+1}{\beta}+i \mu\right)^{2}\right\}\right] \\
& \times \Phi\left(\frac{D-1}{2} ; \frac{i}{2 \rho}\left\{\sigma^{2}+\left(\pi\left(\frac{2 m+1}{\beta}+i \mu\right)^{2}\right\}, 1\right),\right.
\end{aligned}
$$

where $\Phi(z ; s, a)$ is the Lerch transcendental function defined by (D.7). This can be regarded as a probability, per unit space-time volume, for a fermion pair-production from the vacuum by an external $E$ field. Taking the $T=\mu=0$ limit

$$
\lim _{T, \mu \rightarrow 0} \operatorname{Im} V_{\beta, \mu}(\sigma ; \rho)=-\frac{\operatorname{tr}[1] \rho^{D / 2}}{2^{D+1} \pi^{D-1}} e^{-\pi \sigma^{2} / \rho} \Phi\left(\frac{D}{2} ; i \frac{\sigma^{2}}{2 \rho}, 1\right),
$$

we reproduce the known results [3, []

$$
\operatorname{Im} V(\sigma)= \begin{cases}\operatorname{tr}[1] \rho /(8 \pi) \cdot \ln \left(1-e^{-\sigma^{2} \pi / \rho}\right) & \text { for } \quad D=2 \\ -\operatorname{tr}[1] \rho^{3 / 2} /(4 \pi)^{2} \cdot e^{-\pi \sigma^{2} / \rho} \Phi\left(\frac{3}{2} ; i \frac{\sigma^{2}}{2 \rho}, 1\right) & \text { for } \quad D=3 .\end{cases}
$$

One can rewrite (4.3) in the following form using the Lerch transformation (D.9),

$$
\begin{aligned}
\operatorname{Im} V(\sigma)=- & \frac{\operatorname{tr}[1] \rho^{D / 2}}{4(2 \pi)^{D / 2}} \Gamma\left(1-\frac{D}{2}\right)\left[\exp \left[\frac{\pi}{2} i\left(1-\frac{D}{2}\right)\right] \zeta\left(1-\frac{D}{2}, i \frac{\sigma^{2}}{2 \rho}\right)\right. \\
& \left.+\exp \left[-\frac{\pi}{2} i\left(1-\frac{D}{2}\right)\right] \zeta\left(1-\frac{D}{2}, 1-i \frac{\sigma^{2}}{2 \rho}\right)\right] .
\end{aligned}
$$

We shall search for the lowest energy configuration of $\sigma$ solving the critical surface equations derived in sect.2. This ground state solution $\sigma(E)$ minimizes the real part of the effective potential, and the chiral symmetry is broken accordingly. On the contrary, we expect that a strong external field might induce a restoration of chiral symmetry. However, we can not conclude immediately whether or not the chiral symmetry is restored even if the fermion mass vanishes, because the effective potential possesses the imaginary part that represents a decay of "symmetry vacuum" to a fermion pair. Indeed, in the case of $T=\mu=0$, the chiral mass term $\sigma(E)\langle\bar{\psi} \psi\rangle$ does not vanish even when $\sigma(E)=0$ in $D=2$ [3].

Hence we determine the symmetry restoration by the criterion whether the chiral mass term vanishes or not [3, 6]

$$
\lim _{\rho \rightarrow \rho_{c}} \sigma(\rho) \operatorname{Im} V^{\prime}(\sigma(\rho) ; \rho, \beta, \mu)=0,
$$


where we have applied the fact that $\langle\bar{\psi} \psi\rangle$ is related to the $\operatorname{Im} V^{\prime}$ by

$$
\langle\bar{\psi} \psi\rangle_{\sigma=\sigma(\rho)}=i N \operatorname{Im} V^{\prime}(\sigma(\rho) ; \rho, \beta, \mu)-\frac{N}{\lambda} \sigma(\rho),
$$

and $\sigma(\rho)$ denotes a solution of gap equation, i.e., $\operatorname{Re} V^{\prime}=0$. To check this criterion, it is sufficient to see whether or not the $\operatorname{Im} V^{\prime}(\sigma ; \rho, \beta, \mu)$ is finite as $\sigma \rightarrow 0$. From (4.2), we have

$$
\operatorname{Im} V^{\prime}(\sigma ; \rho, \beta, \mu)=\sigma \operatorname{tr}[1] \frac{\rho^{(D-3) / 2}}{2^{D-1} \pi^{D-3} \beta} \sum_{n=1}^{\infty} n^{\frac{3-D}{2}} e^{-\frac{n \pi}{\rho}\left(\sigma^{2}-\mu^{2}\right)} \Theta_{2}\left(\frac{2 n \pi \mu}{\rho \beta}, i \frac{4 n \pi^{2}}{\rho \beta^{2}}\right) .
$$

The upper bound for the summation value in (4.8) can be estimated in the following way

$$
\begin{aligned}
& \sum_{n=1}^{\infty} n^{\frac{3-D}{2}} e^{-\frac{n \pi}{\rho}\left(\sigma^{2}-\mu^{2}\right)} \Theta_{2}\left(\frac{2 n \pi \mu}{\rho \beta}, i \frac{4 n \pi^{2}}{\rho \beta^{2}}\right) \\
= & \sum_{n=1}^{\infty} n^{\frac{3-D}{2}} \sum_{m=-\infty}^{\infty} \exp \left[-n \frac{\pi}{\rho}\left\{\sigma^{2}+\left(\pi \frac{2 m+1}{\beta}-i \mu\right)^{2}\right\}\right] \\
\leq & \int_{0}^{\infty} d x x^{\frac{3-D}{2}} \sum_{m=-\infty}^{\infty} \exp \left[-x \frac{\pi}{\rho}\left\{\sigma^{2}+\left(\pi \frac{2 m+1}{\beta}-i \mu\right)^{2}\right\}\right] .
\end{aligned}
$$

Performing the $x$-integration

$$
\operatorname{RHS}(4.9)=\Gamma\left(\frac{5-D}{2}\right)\left(\frac{\rho}{\pi}\right)^{\frac{5-D}{2}} \sum_{m=-\infty}^{\infty}\left[\sigma^{2}+\left(\pi \frac{2 m+1}{\beta}-i \mu\right)^{2}\right]^{\frac{D-5}{2}},
$$

we find it finite as $\sigma \rightarrow 0$

$$
\lim _{\sigma \rightarrow 0} \operatorname{RHS}(4.9)=2 \Gamma\left(\frac{5-D}{2}\right)\left(\frac{2 \pi^{2}}{\rho \beta}\right)^{\frac{D-5}{2}} \operatorname{Re} \zeta\left(5-D, \frac{1}{2}+i \frac{\beta \mu}{2 \pi}\right) .
$$

The criterion is therefore satisfied

$$
\lim _{\sigma \rightarrow 0} \sigma \operatorname{Im} V^{\prime}(\sigma ; \rho, \beta, \mu)=0
$$

and we conclude that the chiral symmetry is restored for finite $T$ and $\mu$ in $2 \leq D<4$.

Note that RHS of (4.11) vanishes right at a tricritical point, whose necessary condition is (2.41). This is not the case with 2'nd order transitions and suggests that the multicriticality can be mightier than the vacuum instability. As a by-product, we obtain a necessary condition for the tri-criticality on the imaginary part

$$
\lim _{\sigma \rightarrow 0} \frac{1}{\sigma} \operatorname{Im} V^{\prime}(\sigma ; \rho, \beta, \mu)=0,
$$


and it leads to

$$
\frac{1}{\beta} \sum_{n=1}^{\infty} n^{\frac{3-D}{2}} e^{\frac{n \pi \mu^{2}}{\rho}} \Theta_{2}\left(\frac{2 n \pi \mu}{\rho \beta}, i \frac{4 n \pi^{2}}{\rho \beta^{2}}\right)=0 .
$$

It is interesting to note that the powers of $\sigma$ in front of $\operatorname{Im} V^{\prime}$ in (4.12) and (4.13) differ by two from each other. Hence (4.12) and (4.13) may be called a kind of discrete versions of $(2.35$ and $(2.38)$.

In the zero temperature cases, one may derive exact expressions for $\operatorname{Im} V^{\prime}$ from (4.2) by taking the $T, \mu \rightarrow 0$ limit like done in (4.4). Here, we only put a brief statement about their power dependence on $\sigma$ in this limit. Using

$$
\lim _{\beta \rightarrow \infty} \frac{1}{\beta} \sum_{m=-\infty}^{\infty} \exp \left[-x \frac{\pi}{\rho}\left\{\sigma^{2}+\left(\pi \frac{2 m+1}{\beta}\right)^{2}\right\}\right]=e^{-x \frac{\pi}{\rho} \sigma^{2}} \frac{1}{\sqrt{4 \pi x}},
$$

we obtain

$$
\lim _{\beta \rightarrow \infty} \frac{1}{\beta} \operatorname{RHS}(4.9)=\frac{1}{2} \pi^{\frac{D}{2}-3} \rho^{\frac{5-D}{2}} \Gamma\left(2-\frac{D}{2}\right) \sigma^{D-4},
$$

and its power dependence on $\sigma$ exactly coincides with known results [3], 6]

$$
\lim _{T, \mu \rightarrow 0} \operatorname{Im} V^{\prime}(\sigma ; \rho, \beta, \mu) \sim \begin{cases}\sigma^{-1} & \text { for } D=2, \\ \text { const. } & \text { for } D=3 \\ \sigma^{1-\epsilon} & \text { for } D=4-\epsilon\end{cases}
$$

Namely, chiral symmetry is restored in $D=3$ [6] and $4-\epsilon$, and broken in $D=2$ [3].

\subsection{The duality and phase structures}

In this subsection, we present phase diagrams with analyzing the real parts of the critical equations (2.36) and (2.39). Also, we point out a dual relation between the electric and magnetic dominant effective potentials (at $T=0$ ) under the rotation $\xi \rightarrow i \rho$. We follow the same method employed in [3, 6] in order to evaluate the real parts (principal values).

Let us introduce the following compact notations for the real part effective potential (2.30) (to avoid lengthy expressions),

$$
\operatorname{Re} V_{R}(\sigma ; \rho, \beta, \mu)=\frac{1}{2 \lambda_{R}} \sigma^{2}+\frac{\operatorname{tr}[1]}{2(4 \pi)^{D / 2}}\left(I_{1}(\sigma)-I_{1}(0)\right),
$$


where

$$
I_{1}(\sigma)=\mathrm{P} \int_{0}^{\infty} \frac{d s}{s^{D / 2}} s \rho \cot s \rho \cdot f(s, \beta, \mu),
$$

and

$$
f(s, \beta, \mu)=\frac{1}{s} e^{-s \sigma^{2}} e^{s \mu^{2}} \frac{\sqrt{4 \pi s}}{\beta} \Theta_{2}\left(s \frac{2 \mu}{\beta}, i s \frac{4 \pi}{\beta^{2}}\right)+\sigma^{2} e^{-s}(1-2 s) .
$$

Furthermore, split $I_{1}(\sigma)=A(\sigma)+B(\sigma)$ into the following two pieces for convenience:

$$
\begin{aligned}
A(\sigma) & =\rho^{D / 2-1} \int_{0}^{\pi / 2} \frac{d \tau}{(4 \pi)^{D / 2}} \tau^{1-D / 2} \cot \tau \cdot f\left(\frac{\tau}{\rho}, \beta, \mu\right) \\
B(\sigma) & =\rho^{D / 2-1} \sum_{n=1}^{\infty} \mathrm{P} \int_{n \pi-\pi / 2}^{n \pi+\pi / 2} \frac{d \tau}{(4 \pi)^{D / 2}} \tau^{1-D / 2} \cot \tau \cdot f\left(\frac{\tau}{\rho}, \beta, \mu\right), \\
& =\rho^{D / 2-1} \sum_{n=1}^{\infty} \int_{0}^{\pi / 2} \frac{d \tau}{(4 \pi)^{D / 2}} \cot \tau \cdot\left[\frac{f\left(\frac{\tau+n \pi}{\rho}, \beta, \mu\right)}{(\tau+n \pi)^{D / 2-1}}-(\tau \rightarrow-\tau)\right] .
\end{aligned}
$$

These quantities $A$ and $B$ have another expressions. Namely, the integration in (4.21) can be performed

$$
\begin{aligned}
A(\sigma)=- & 2 \sum_{k=0}^{\infty} \zeta(2 k)\left(\frac{\rho}{\pi}\right)^{2 k}\left[2 \frac{\sqrt{4 \pi}}{\beta} \operatorname{Re} \sum_{m=0}^{\infty}\left[\sigma^{2}+\left(\pi \frac{2 m+1}{\beta}+i \mu\right)^{2}\right]^{-2 k+\frac{D-1}{2}}\right. \\
& \times \gamma\left(\frac{1-D}{2}+2 k, \frac{\pi}{2 \rho}\left\{\sigma^{2}+\left(\pi \frac{2 m+1}{\beta}+i \mu\right)^{2}\right\}\right) \\
& \left.+\sigma^{2} \gamma\left(1-\frac{D}{2}+2 k, \frac{\pi}{2 \rho}\right)-2 \sigma^{2} \gamma\left(2-\frac{D}{2}+2 k, \frac{\pi}{2 \rho}\right)\right] .
\end{aligned}
$$

Here we have applied the formula

$$
\int_{0}^{\pi / 2} d x x^{z} e^{-\alpha x} \cot x=-2 \sum_{k=0}^{\infty} \frac{\zeta(2 k)}{\pi^{2 k}} \alpha^{-2 k-z} \gamma\left(z+2 k, \frac{\pi}{2} \alpha\right),
$$

where $\zeta(z)$ is the zeta function, and $\gamma(z, p)$ the incomplete gamma function defined by

$$
\gamma(z, p)=\int_{0}^{p} e^{-t} t^{z-1} d t \quad \operatorname{Re} z>0 .
$$

The summation of the second line in (4.22) can be performed

$$
\begin{aligned}
B(\sigma)=\int_{0}^{\pi / 2} d \tau \cot \tau \cdot\left[\frac{\sqrt{4 \pi}}{\beta} 2 \operatorname{Re} \sum_{m=0}^{\infty} \exp \left[-\frac{\tau+\pi}{\rho}\left\{\sigma^{2}+\left(\pi \frac{2 m+1}{\beta}+i \mu\right)^{2}\right\}\right]\right. \\
\quad \times\left(\frac{\rho}{\pi}\right)^{\frac{D-1}{2}} \Phi\left(\frac{D-1}{2} ; \frac{i}{2 \rho}\left\{\sigma^{2}+\left(\pi \frac{2 m+1}{\beta}+i \mu\right)^{2}\right\}, 1+\frac{\tau}{\pi}\right) \\
+\sigma^{2}\left(\frac{\rho}{\pi}\right)^{\frac{D}{2}-1} e^{-(\tau+\pi) / \rho} \Phi\left(\frac{D}{2}-1 ; \frac{i}{2 \rho}, 1+\frac{\tau}{\pi}\right) \\
\left.\quad-2 \sigma^{2}\left(\frac{\rho}{\pi}\right)^{\frac{D}{2}-2} e^{-(\tau+\pi) / \rho} \Phi\left(\frac{D}{2}-2 ; \frac{i}{2 \rho}, 1+\frac{\tau}{\pi}\right)-(\tau \rightarrow-\tau)\right] .
\end{aligned}
$$


Similarly, we rewrite the second and third order critical equations (2.36) and (2.39) in a straightforward way,

$$
\begin{gathered}
I_{2}(\sigma)+2 \Gamma\left(2-\frac{D}{2}\right)=0 \\
I_{3}(\sigma)=0
\end{gathered}
$$

where the explicit forms of $I_{2}$ and $I_{3}$ are given in Appendix D.

For simplicity, let us begin with analyzing the $T=\mu=0$ case. In this case, (4.20) simplifies because of (2.22) (If one wants to consider the $\mu \neq 0$ situation, it is necessary to follow the similar procedure analyzed in sect.3, and see also [27]). Let us write down $I_{1}$ and $I_{2}$ :

$$
\begin{aligned}
I_{1}(\sigma)= & \rho^{D / 2-1} \int_{0}^{\pi / 2} d \tau \cot \tau \cdot\left[\rho \tau^{-D / 2} e^{-\tau \sigma^{2} / \rho}+\sigma^{2} \tau^{1-D / 2} e^{-\tau / \rho}\left(1-\frac{2 \tau}{\rho}\right)\right. \\
& +\pi^{-D / 2}\left\{\rho e^{-(\tau+\pi) \sigma^{2} / \rho} \Phi\left(\frac{D}{2} ; i \frac{\sigma^{2}}{2 \rho}, 1+\frac{\tau}{\pi}\right)\right. \\
& +\sigma^{2} \pi e^{-(\tau+\pi) / \rho} \Phi\left(\frac{D}{2}-1 ; \frac{i}{2 \rho}, 1+\frac{\tau}{\pi}\right) \\
& \left.\left.-\sigma^{2} \frac{2 \pi^{2}}{\rho} e^{-(\tau+\pi) / \rho} \Phi\left(\frac{D}{2}-2 ; \frac{i}{2 \rho}, 1+\frac{\tau}{\pi}\right)-(\tau \rightarrow-\tau)\right\}\right], \\
I_{1}(0)= & \rho^{\frac{D}{2}} \int_{0}^{\pi / 2} d \tau \cot \tau \cdot\left[\tau^{\frac{-D}{2}}+\pi^{\frac{-D}{2}}\left\{\zeta\left(\frac{D}{2}, 1+\frac{\tau}{\pi}\right)-\zeta\left(\frac{D}{2}, 1-\frac{\tau}{\pi}\right)\right\}\right],
\end{aligned}
$$

and differentiating $I_{1}$,

$$
\begin{aligned}
I_{2}(\sigma)= & \left.\frac{\partial}{\partial \sigma^{2}} I_{1}(\sigma)\right|_{\sigma=0} \\
= & \rho^{D / 2-1} \int_{0}^{\pi / 2} d \tau \cot \tau \cdot\left[\tau^{1-D / 2}\left\{-1+e^{-\tau / \rho}\left(1-\frac{2 \tau}{\rho}\right)\right\}\right. \\
& +\pi^{1-D / 2}\left\{-\zeta\left(\frac{D}{2}-1,1+\frac{\tau}{\pi}\right)+e^{-(\tau+\pi) / \rho} \Phi\left(\frac{D}{2}-1 ; \frac{i}{2 \rho}, 1+\frac{\tau}{\pi}\right)\right. \\
& \left.\left.-\frac{2 \pi}{\rho} e^{-(\tau+\pi) / \rho} \Phi\left(\frac{D}{2}-2 ; \frac{i}{2 \rho}, 1+\frac{\tau}{\pi}\right)-(\tau \rightarrow-\tau)\right\}\right] .
\end{aligned}
$$

We obtain the following critical $\rho$-values as the solutions of (4.27) with (4.31)

$$
\rho_{c}=\left\{\begin{array}{lll}
0.583 & \text { for } \quad D=2 \\
1.238 & \text { for } \quad D=3 \\
3.394 & \text { for } \quad D=3.5
\end{array}\right.
$$


and we verified that the real part of the potential given by 4.18) with (4.29) and (4.30) behaves consistently. Here we add a few remarks on these results. From $D=2$ to $D=3.3$, whose $\rho_{c}=1.965$, all $\rho_{c}$-values excellently coincide with the solutions of the following analytic equation

$$
\begin{aligned}
0= & 2-(2 \rho)^{\frac{D}{2}-1} \zeta\left(2-\frac{D}{2}\right) \cos \left[\frac{\pi}{4}(D-2)\right] \\
& +\operatorname{Re}\left[(2 i \rho)^{\frac{D}{2}-1} \zeta\left(2-\frac{D}{2}, 1-\frac{i}{2 \rho}\right)+(2 i \rho)^{\frac{D}{2}-2}(D-4) \zeta\left(3-\frac{D}{2}, 1-\frac{i}{2 \rho}\right)\right],
\end{aligned}
$$

and this still exhibits a good agreement 9 at $D=3.5\left(\rho_{c}=3.395\right)$. We found this equation from (3.30) through rotating $\xi \rightarrow i \rho$, taking real part and the limit $m \rightarrow 0$. The divergence stemming from $m \rightarrow 0$ now drops off since it becomes a pure imaginary number by the rotation. Similarly, we verify that those critical values are consistent with the following potential derived by the same rotation procedure from (3.8);

$$
\begin{aligned}
V(\sigma ; \rho)=\frac{\sigma^{2}}{2 \lambda_{R}} & +\operatorname{Re}\left[\frac { \operatorname { t r } [ 1 ] i \rho } { 2 ( 4 \pi ) ^ { D / 2 } } \left[2 \sigma^{2}(2 i \rho)^{\frac{D}{2}-2} \zeta\left(2-\frac{D}{2}, 1-\frac{i}{2 \rho}\right) \Gamma\left(2-\frac{D}{2}\right)\right.\right. \\
& -4 \sigma^{2}(2 i \rho)^{\frac{D}{2}-3} \zeta\left(3-\frac{D}{2}, 1-\frac{i}{2 \rho}\right) \Gamma\left(3-\frac{D}{2}\right) \\
& \left.\left.+2(2 i \rho)^{\frac{D}{2}-1} \Gamma\left(1-\frac{D}{2}\right)\left\{\zeta\left(1-\frac{D}{2}, 1-\frac{i \sigma^{2}}{2 \rho}\right)-\zeta\left(1-\frac{D}{2}\right)\right\}\right]\right] .
\end{aligned}
$$

Note that this rotation procedure is not always allowed in general cases (for example in the thermodynamic part $\tilde{V}$ ), because we have used the expansion (3.6) (or the formula (3.26) derived from (3.6)), which is not valid for a complex analytic function. In this sense, the above coincidences are non-trivial results (although they are rather trivial before the expansion is applied).

Now we discuss the phase structures in the $\rho-T$ - $\mu$ space. In Fig.9, we depict phase diagrams for several $\mu$ values in the case of $D=2$. As mentioned above, the vacuum state is not invariant at $T=\mu=0$. In this sense, the critical value $\rho_{c}$ divides a phase between massive states (broken phase) and quasi-stational states (quasi-symmetric phase), and the total probability of decay per volume is given by [3]

$$
\Gamma=-\lim _{\rho \rightarrow \rho_{c}} 2 \sigma \operatorname{Im} V^{\prime}(\sigma ; \rho, \infty, 0)=\frac{\rho_{c}}{\pi} .
$$

${ }^{6}$ The deviation of $\rho_{c}$ grows as $D$ increasing in the region $D>3.5$. We believe this is related to a singular problem in numerical computations like encountered in sect. 3.2. 
The similar situation is expected for finite $\mu$ (with $T=0$ ) as well, and the $\Gamma$ changes into $\Gamma+\Delta \Gamma$, where $\Delta \Gamma$ is formally given by

$$
\Delta \Gamma=-\lim _{\rho \rightarrow \rho_{c}} 2 \sigma \operatorname{Im} \tilde{V}^{\prime}(\sigma ; \rho, \infty, \mu)
$$

although we have not estimated its explicit value yet. On the other hand, as discussed previously, the chiral symmetry can be restored (i.e., $\Gamma=0$ ) when both of $T$ and $\mu$ are finite.

In Figs. 10 and 11, the cases of $D=3$ and $4-\epsilon(\epsilon=0.5)$ are illustrated. As shown in (4.17), these cases have a stable (chiral symmetric) vacuum at $T=\mu=0$. Also, in the situation of finite $T$ and $\mu$, the chiral symmetry is restored similarly to the $D=2$ case. In each dimension, the critical surface goes down to a low temperature region as $\mu$ increasing. We could not find any tricritical point within a region of significant numerical data. In particular the computation did not work well below around $T \cong 0.1$, and we hence extrapolate the critical lines in Figs.9-11.

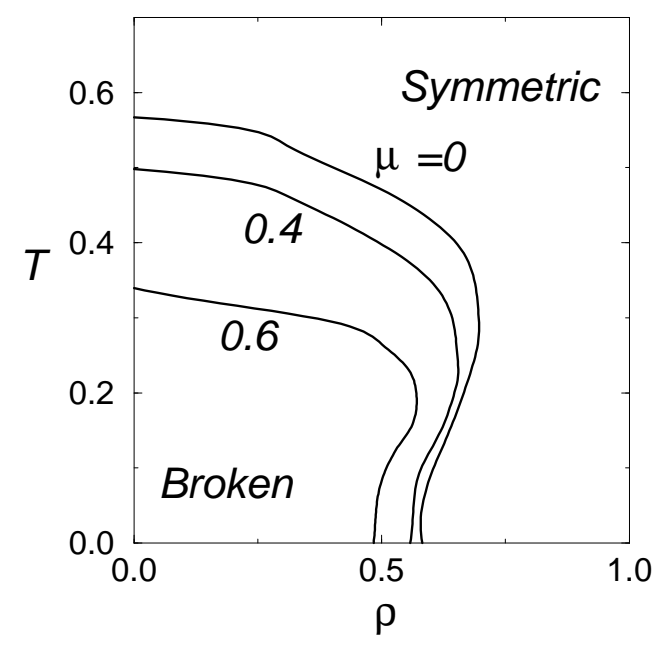

Fig. 9: The critical lines for electric dominant cases in $D=2$.

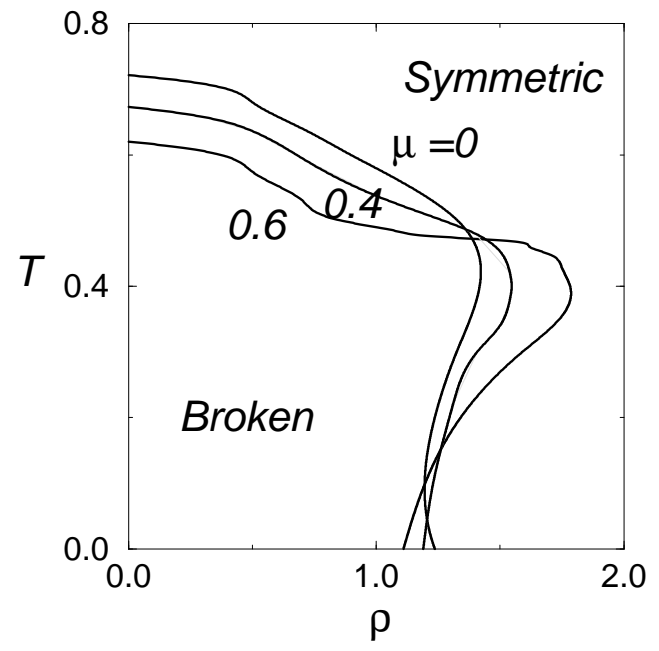

Fig. 10: The critical lines for electric dominant cases in $D=3$. 


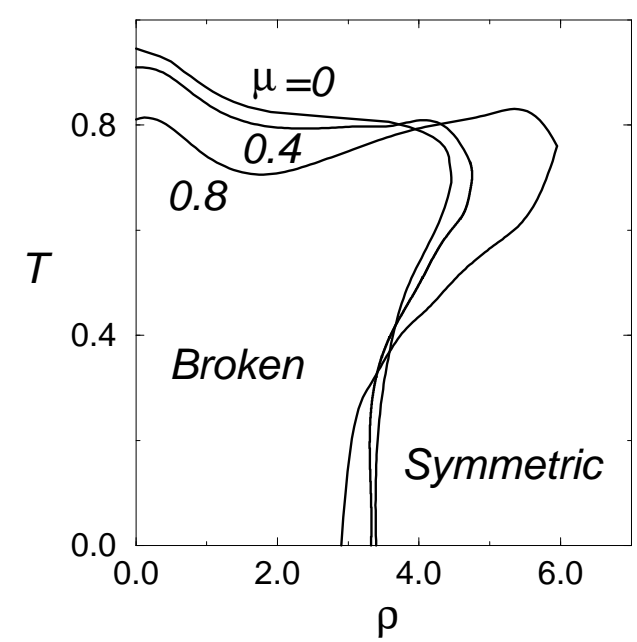

Fig. 11: The critical lines for electric dominant cases in $D=4-\epsilon$, where $\epsilon$ is chosen to be 0.5 .

\section{Conclusion and discussion}

In this paper, we found various interesting properties of the thermodynamic effective potential of the large- $N$ Gross-Neveu model coupled to constant electromagnetic fields perpendicular to each other: worldline approach, magnetic discretization, electromagnetic duality, critical equations and chiral phase structures.

First, we summarize the analytic properties of the effective potentials. In sect.2, we presented a new derivation of the effective potential from the point of the worldline approach developed in recent years [22, 23]. The similar formulation had been applied to constant curvature cases in [17], but we could not clarify the correspondence to other calculations. However, we emphasize that it is interesting that our method proposed in sect.2 leads to the exact results [11, 24]. Another spectacular of our potential can be seen in sect.3. Our potential reveals the role of $\xi$ as a discrete momentum unit even in the situations under finite temperature with keeping $\mu$ finite, and in particular, the discrete potential of $D=3$ completely resembles to the continuum potential [19]. In other words, our $D$-dimensional thermodynamic effective potential is composed of the sum over discrete energy spectra $\epsilon_{n}= \pm \sqrt{m^{2}+2 n \xi}, n \in Z$. We also argued how to take the $\beta \rightarrow \infty$ limit 
of the potential with $\xi$ and $\mu$ kept finite, and we described the precise continuum limit $\xi \rightarrow 0$, which gives rise to the known results of $\mu_{c}$. It means $\mu$ is properly taken into account in our derivation.

We found in sect.4 the tri-criticality condition for the imaginary part of the electric dominant effective potential, and also found the analytic expressions (4.33) and (4.34) which are dual to the magnetic dominant effective potential and its gap equation at $T=$ $\mu=0$. When the effective potential possesses the imaginary part, the vanishing conditions on the first and second derivatives of $\operatorname{Re} V$ are not sufficient for defining second order critical and tricritical points. It is necessary for second order critical points to satisfy the condition (4.12), which is a vanishing condition on the chiral mass term $\sigma \operatorname{Im} V^{\prime}$. Similarly, (4.13), $\sigma^{-1} \operatorname{Im} V^{\prime}=0$, should be satisfied at a tricritical point as well. These vanishing conditions on $\operatorname{Im} V^{\prime}$ may be regarded as a discrete version of the real part conditions (2.35) and (2.38).

Based on all these properties, we investigated the phase structures in various cases. First, in sect.3, we showed the phase diagrams for magnetic dominant cases, and observed the strong catalyst effects of magnetic fields. When $\beta=\infty(\mu \neq 0$ in $D=3$ and 3.5), the symmetry restorations are not the second but the first order. In these cases, the chiral symmetry becomes broken in a strong magnetic field region. This property is the same for finite $\beta$ (with $\mu \neq 0$ ) also. These features do not change if we formally extend to the $2<D<3$ regions, from which we actually got some insights toward a region $D$ being near to 4 . As pointed out in [28], the effective potential of $D=2$ by itself resembles to an electrostatic potential problem of the charge distribution $s \operatorname{coth} s$ along a one-dimensional line. It means that such an electric distribution rather acts as a "magnetic" catalyst, if we could realize the distribution in a one-dimensional laboratory.

The phase diagram analyses for the electric dominant cases are more complicated. First we have to test whether the imaginary part of the effective potential vanishes as $\sigma$ goes to zero (in other words, the chiral mass term $\sigma\langle\bar{\psi} \psi\rangle$ vanishes or not, q.v. (4.6)). At $T=\mu=0$, this can be easily checked by integrating (4.17) w.r.t. $\sigma$, and it shows that the $\operatorname{Im} V(\sigma)$ behaves like $\sigma^{D-2}$. For $2<D<4$, the chiral symmetry gets restored. For $D=2$, the $\operatorname{Im} V^{\prime}$ diverges as $\sigma \rightarrow 0$, and the imaginary part $\operatorname{Im} V$ never vanishes [3].

However, at finite $\beta$ and $\mu$, we showed that the chiral symmetry is restored in $2 \leq$ 
$D<4$ based on the same criterion. This suggests that a symmetrization effect by high temperature can be stronger than the vacuum instability by an external field. As to $\rho-\mu$ plane, we have not yet succeeded in formulating the $T=0$ limit with $\mu$ being kept finite (In this sense, the achievement of the magnetic case was remarkable). When the fermion density is large, a density effect might enhance the chiral symmetry, and consequently, the instability would be suppressed. In order to address this question (at $T=0$ ), it is necessary to study the 1'st order critical equation like done in (3.28). An interesting problem is whether or not a dual relation like the one between (3.30) and (4.33) holds in this case, because it contains a non-trivial part, the thermodynamic quantity $\tilde{V}$, which has non-analytic dependence on $\mu$.

\section{A A proof of (3.14)}

First, we show how (2.36) and (2.39) reproduce (2.40) and (2.41) respectively. Then we present a proof of (3.14). In the limit $\xi \rightarrow 0$, (2.36) becomes (2.40) in the following way

$$
\begin{aligned}
0 & =\int_{0}^{\infty} d s s^{-D / 2}\left[e^{-s}-e^{s \mu^{2}} \frac{\sqrt{4 \pi s}}{\beta} \Theta_{2}\left(s \frac{2 \mu}{\beta}, i s \frac{4 \pi}{\beta^{2}}\right)\right] \\
& =\Gamma\left(1-\frac{D}{2}\right)-2 \frac{\sqrt{4 \pi}}{\beta} \int_{0}^{\infty} d s s^{\frac{1-D}{2}} \operatorname{Re} \sum_{n=1}^{\infty} \exp \left[-s \frac{4 \pi^{2}}{\beta^{2}}\left(n-\frac{1}{2}+i \frac{\beta \mu}{2 \pi}\right)^{2}\right] \\
& =\Gamma\left(1-\frac{D}{2}\right)-2 \frac{\sqrt{4 \pi}}{\beta}\left(\frac{2 \pi}{\beta}\right)^{D-3} \Gamma\left(\frac{3-D}{2}\right) \operatorname{Re} \sum_{n=0}^{\infty}\left(n+\frac{1}{2}+i \frac{\beta \mu}{2 \pi}\right)^{D-3} \\
& =\Gamma\left(1-\frac{D}{2}\right)-\frac{2}{\sqrt{\pi}}\left(\frac{2 \pi}{\beta}\right)^{D-2} \Gamma\left(\frac{3-D}{2}\right) \operatorname{Re} \zeta\left(3-D, \frac{1}{2}+i \frac{\beta \mu}{2 \pi}\right),
\end{aligned}
$$

and similarly $(2.39)$ reads

$$
\begin{aligned}
0 & =\int_{0}^{\infty} d s s^{1-\frac{D}{2}} e^{s \mu^{2}} \frac{\sqrt{4 \pi s}}{\beta} \Theta_{2}\left(s \frac{2 \mu}{\beta}, i s \frac{4 \pi}{\beta^{2}}\right) \\
& =\frac{2}{\sqrt{\pi}}\left(\frac{2 \pi}{\beta}\right)^{D-4} \Gamma\left(\frac{5-D}{2}\right) \operatorname{Re} \zeta\left(5-D, \frac{1}{2}+i \frac{\beta \mu}{2 \pi}\right) .
\end{aligned}
$$

Let us turn to the proof of (3.14) making use of the above calculation. Recalling the 
definition of $\mathcal{O}_{\beta}(\sigma)$ by (3.10), and using (2.24), we have

$$
\mathcal{O}_{\beta}(\sigma)=\int_{0}^{\infty} d s s^{-D / 2} e^{-s \sigma^{2}}\left[\Theta_{4}\left(i \frac{\beta \mu}{2 \pi}, i \frac{\beta^{2}}{4 \pi s}\right)-1\right] .
$$

The top line of (A.1) can also be written in the form

$$
0=\int_{0}^{\infty} d s s^{-D / 2}\left[e^{-s}-\Theta_{4}\left(i \frac{\beta \mu}{2 \pi}, i \frac{\beta^{2}}{4 \pi s}\right)\right] .
$$

If $2<D<4$, the following integral is convergent

$$
\int_{0}^{\infty} d s s^{-D / 2}\left(e^{-s}-1\right)=\frac{1}{1-\frac{D}{2}} \int_{0}^{\infty} d s s^{1-\frac{D}{2}} e^{-s}=\Gamma\left(1-\frac{D}{2}\right),
$$

and therefore (A.3) amounts to

$$
0=\Gamma\left(1-\frac{D}{2}\right)-\mathcal{O}_{\beta}(0)
$$

Comparing the RHS of this equation with the bottom line of (A.1), we have proven (3.14).

\section{B The $\xi \rightarrow 0$ limit of (3.22)}

We prove that $(3.22)$ is reduced to $(3.19)$ in the limit $\xi \rightarrow 0$. Obviously, the 1 'st term $\mathcal{O}_{\infty}(0)$ in $(3.22)$ drops in $\xi \rightarrow 0$, since $\mathcal{O}_{\infty}(0)$ possesses no dependence on $\xi$. Hence, we have only to prove the following equality

$$
\lim _{\xi \rightarrow 0} \sum_{l=1}^{L} \xi \int_{0}^{\mu-\sqrt{2 l \xi}}\left(t^{2}+2 t \sqrt{2 l \xi}\right)^{\frac{D-3}{2}} d t=\frac{\mu^{D}}{D(D-1)} .
$$

Under the correspondence (3.18), LHS of the above equation can be transformed into

$$
\begin{aligned}
\operatorname{LHS}(B .1) & =\int_{0}^{\mu} k d k \int_{0}^{\mu-k}\left(t^{2}+2 k t\right)^{\frac{D-3}{2}} d t=\int_{0}^{\mu} d t \int_{0}^{\mu-t} k\left(t^{2}+2 k t\right)^{\frac{D-3}{2}} d k \\
& =\int_{0}^{\mu} d t t^{D-1} \int_{0}^{(\mu-t) / t} k(1+2 k)^{\frac{D-3}{2}} d k
\end{aligned}
$$

and applying the formula 3.194.1 [29]

$$
\int_{0}^{u} x^{\mu-1}(1+\beta x)^{-\nu} d x=\frac{1}{\mu} u^{\mu} F(\nu, \mu ; 1+\mu ;-\beta u), \quad|\arg (1+\beta u)|<\pi, \quad \operatorname{Re} \mu>0,
$$

we get

$$
\operatorname{LHS}(B .1)=\frac{1}{2} \int_{0}^{\mu} t^{D-3}(\mu-t)^{2} F\left(\frac{3-D}{2}, 2 ; 3 ;-2 \frac{\mu-t}{t}\right) .
$$


Now changing the integration variable, $t=2 \mu /(w+2)$,

$$
\operatorname{LHS}(B .1)=\mu^{D} 2^{D-3} \int_{0}^{\infty} d w w^{2}(w+2)^{-1-D} F\left(\frac{3-D}{2}, 2 ; 3 ;-w\right)
$$

and applying the formula 7.512.10 29]

$$
\int_{0}^{\infty} x^{\gamma-1}(x+z)^{-\sigma} F(\alpha, \beta ; \gamma ;-x) d x=\frac{\Gamma(\gamma) \Gamma(\alpha-\gamma+\sigma) \Gamma(\beta-\gamma+\sigma)}{\Gamma(\sigma) \Gamma(\alpha+\beta-\gamma+\sigma)},
$$

(where $\operatorname{Re} \gamma>0, \operatorname{Re} \alpha-\gamma+\sigma>0, \operatorname{Re} \beta-\gamma+\sigma>0,|\arg z|<\pi$ ), we find

$$
\text { LHS }(B .1)=\mu^{D} 2^{D-3} \frac{\Gamma(3) \Gamma\left(\frac{D-1}{2}\right) \Gamma(D)}{\Gamma(D+1) \Gamma\left(\frac{D+3}{2}\right)} F\left(\frac{D-1}{2}, D ; \frac{D+3}{2} ;-1\right) .
$$

Using a Kummer transformation to make the argument of hypergeometric function from -1 to $1 / 2$ and the following formula

$$
F\left(\alpha, 1-\alpha ; \gamma ; \frac{1}{2}\right)=\frac{2^{1-\gamma} \Gamma(\gamma) \sqrt{\pi}}{\Gamma\left(\frac{\alpha+\gamma}{2}\right) \Gamma\left(\frac{\gamma-\alpha+1}{2}\right)},
$$

we have

$$
F\left(\frac{D-1}{2}, D ; \frac{3+D}{2} ;-1\right)=\frac{2^{1-D} \Gamma\left(\frac{3+D}{2}\right)}{\Gamma\left(\frac{D+1}{2}\right)} .
$$

Finally substituting this into $(\mathbb{B} .7)$, the proof ends

$$
\operatorname{LHS}(B .1)=\mu^{D} \frac{1}{4} \frac{\Gamma(3) \Gamma(D) \Gamma\left(\frac{D-1}{2}\right)}{\Gamma(D+1) \Gamma\left(\frac{D+1}{2}\right)}=\frac{\mu^{D}}{D(D-1)} .
$$

\section{The $\xi \rightarrow 0$ limit of (3.28)}

We prove (3.28) goes to (3.31) in the limit $\xi \rightarrow 0$. Eq.(3.28) is nothing but the sum of two quantities

$$
0=V(m ; \xi)+\tilde{V}(m ; \xi, \infty, \mu)
$$

where $V(m, \xi)$ is given by (3.27), and $\tilde{V}(m ; \xi, \infty, \mu)$ is given by RHS of (3.22). According to the result of Appendix B, we have

$$
\lim _{\xi \rightarrow 0} \tilde{V}(m ; \xi, \infty, \mu)=\frac{\operatorname{tr}[1] 2 \sqrt{\pi}}{(4 \pi)^{D / 2} \Gamma\left(\frac{D-1}{2}\right)} \frac{\mu^{D}}{D(D-1)} .
$$

Let us consider the limit of another quantity $V(m ; \xi)$. In the situation of $\xi \rightarrow 0$, three terms of zeta function on RHS of (3.27) only survive

$$
\begin{aligned}
V(m ; \xi) \cong & \frac{\operatorname{tr}[1] \xi}{2(4 \pi)^{D / 2}}\left[2(2 \xi)^{\frac{D}{2}-2} m^{2} \Gamma\left(2-\frac{D}{2}\right) \sum_{n=0}^{\infty} \frac{1}{\left(n+1+m^{2} / 2 \xi\right)^{2-D / 2}}\right. \\
& \left.+2(2 \xi)^{\frac{D}{2}-1} \Gamma\left(1-\frac{D}{2}\right) \sum_{n=0}^{\infty}\left\{\frac{1}{\left(n+1+m^{2} / 2 \xi\right)^{1-D / 2}}-\frac{1}{(n+1)^{1-D / 2}}\right\}\right]
\end{aligned}
$$


and we adopt the following continuum limit as $\xi \rightarrow 0$,

$$
(n+1) 2 \xi \quad \rightarrow \quad x, \quad 2 \xi \sum_{n=0}^{\infty} \rightarrow \int_{0}^{\infty} d x
$$

The limit of (C.3) is therefore led to the following

$$
\begin{aligned}
\lim _{\xi \rightarrow 0} V(m ; \xi) & =\frac{\operatorname{tr}[1]}{2(4 \pi)^{D / 2}} \int_{0}^{\infty} d x\left[\frac{-m^{2} \Gamma\left(2-\frac{D}{2}\right)}{\left(x+m^{2}\right)^{2-D / 2}}+\Gamma\left(1-\frac{D}{2}\right)\left\{\left(x+m^{2}\right)^{\frac{D}{2}-1}-x^{\frac{D}{2}-1}\right\}\right] \\
& =\left.\frac{\operatorname{tr}[1]}{2(4 \pi)^{D / 2}} \Gamma\left(1-\frac{D}{2}\right)\left[\left(x+m^{2}\right)^{D / 2}\left(\frac{2}{D}-\frac{m^{2}}{x+m^{2}}\right)-\frac{2}{D} x^{D / 2}\right]\right|_{0} ^{\infty} \\
& =\frac{\operatorname{tr}[1]}{2(4 \pi)^{D / 2}} \Gamma\left(1-\frac{D}{2}\right)\left(1-\frac{2}{D}\right) m^{D}
\end{aligned}
$$

Combining (C.2) and (C.5), the limit of (C.1) turns out to be

$$
\frac{1}{2} \Gamma\left(1-\frac{D}{2}\right)\left(1-\frac{2}{D}\right) m^{D}+\frac{2 \sqrt{\pi}}{\Gamma\left(\frac{D-1}{2}\right)} \frac{\mu^{D}}{D(D-1)}=0 \text {. }
$$

This is equivalent to (3.31), and the proof ends.

\section{The explicit forms of $I_{2}(\sigma)$ and $I_{3}(\sigma)$}

We write down explicit expressions for $I_{2}(\sigma)$ and $I_{3}(\sigma)$, which are defined by

$$
\begin{aligned}
& I_{2}(\sigma)=\left.\frac{\partial}{\partial \sigma^{2}} I_{1}(\sigma)\right|_{\sigma=0}, \\
& I_{3}(\sigma)=\left.\frac{\partial^{2}}{\partial\left(\sigma^{2}\right)^{2}} I_{1}(\sigma)\right|_{\sigma=0} .
\end{aligned}
$$

$I_{2}(\sigma)$ is composed of the sum of the following two quantities:

$$
\begin{aligned}
\left.\frac{\partial}{\partial \sigma^{2}} A(\sigma)\right|_{\sigma=0}=2 & \sum_{k=0}^{\infty} \zeta(2 k)\left(\frac{\rho}{\pi}\right)^{2 k}\left[2 \frac{\sqrt{4 \pi}}{\beta} \operatorname{Re} \sum_{m=0}^{\infty}\left(\pi \frac{2 m+1}{\beta}+i \mu\right)^{D-3-4 k}\right. \\
& \times \gamma\left(\frac{3-D}{2}+2 k, \frac{\pi}{2 \rho}\left(\pi \frac{2 m+1}{\beta}+i \mu\right)^{2}\right) \\
& \left.-\gamma\left(1-\frac{D}{2}+2 k, \frac{\pi}{2 \rho}\right)+2 \gamma\left(2-\frac{D}{2}+2 k, \frac{\pi}{2 \rho}\right)\right],
\end{aligned}
$$

and

$$
\left.\frac{\partial}{\partial \sigma^{2}} B(\sigma)\right|_{\sigma=0}=\int_{0}^{\pi / 2} d \tau \cot \tau \cdot\left[-2 \frac{\sqrt{4 \pi}}{\beta}\left(\frac{\rho}{\pi}\right)^{\frac{D-3}{2}} \operatorname{Re} \sum_{m=0}^{\infty} \exp \left[-\frac{\tau+\pi}{\rho}\left(\pi \frac{2 m+1}{\beta}+i \mu\right)^{2}\right]\right.
$$




$$
\begin{aligned}
& \times \Phi\left(\frac{D-3}{2} ; \frac{i}{2 \rho}\left(\pi \frac{2 m+1}{\beta}+i \mu\right)^{2}, 1+\frac{\tau}{\pi}\right) \\
& +\left(\frac{\rho}{\pi}\right)^{\frac{D}{2}-1} e^{-(\tau+\pi) / \rho} \Phi\left(\frac{D}{2}-1 ; \frac{i}{2 \rho}, 1+\frac{\tau}{\pi}\right) \\
& \left.-2\left(\frac{\rho}{\pi}\right)^{\frac{D}{2}-2} e^{-(\tau+\pi) / \rho} \Phi\left(\frac{D}{2}-2 ; \frac{i}{2 \rho}, 1+\frac{\tau}{\pi}\right)-(\tau \rightarrow-\tau)\right]
\end{aligned}
$$

And similarly, $I_{3}(\sigma)$ is composed of:

$$
\begin{aligned}
\left.\frac{\partial^{2}}{\partial\left(\sigma^{2}\right)^{2}} A(\sigma)\right|_{\sigma=0}=- & 4 \frac{\sqrt{4 \pi}}{\beta} \sum_{k=0}^{\infty} \zeta(2 k)\left(\frac{\rho}{\pi}\right)^{2 k} \operatorname{Re} \sum_{m=0}^{\infty}\left(\pi \frac{2 m+1}{\beta}+i \mu\right)^{D-5-4 k} \\
& \times \gamma\left(\frac{5-D}{2}+2 k, \frac{\pi}{2 \rho}\left(\pi \frac{2 m+1}{\beta}+i \mu\right)^{2}\right)
\end{aligned}
$$

and

$$
\begin{gathered}
\left.\frac{\partial^{2}}{\partial\left(\sigma^{2}\right)^{2}} B(\sigma)\right|_{\sigma=0}=2 \frac{\sqrt{4 \pi}}{\beta}\left(\frac{\rho}{\pi}\right)^{\frac{D-5}{2}} \int_{0}^{\pi / 2} d \tau \cot \tau \cdot \operatorname{Re}\left[\sum_{m=0}^{\infty} \exp \left[-\frac{\tau+\pi}{\rho}\left(\pi \frac{2 m+1}{\beta}+i \mu\right)^{2}\right]\right. \\
\left.\times \Phi\left(\frac{D-5}{2} ; \frac{i}{2 \rho}\left(\pi \frac{2 m+1}{\beta}+i \mu\right)^{2}, 1+\frac{\tau}{\pi}\right)-(\tau \rightarrow-\tau)\right]
\end{gathered}
$$

Our notation for the Lerch transcendental $\Phi(z ; s, a)$ is

$$
\Phi(z ; s, a)=\sum_{n=0}^{\infty} \frac{e^{2 \pi i n s}}{(n+a)^{z}}, \quad \operatorname{Re} z>1
$$

and a Lerch transformation is then expressed by

$$
\begin{aligned}
(2 \pi)^{z} \Phi(1-z ; s, a)= & \Gamma(z)\left[\exp \left[\pi i\left(\frac{z}{2}-2 a s\right)\right] \Phi(z ;-a, s)\right. \\
& \left.+\exp \left[\pi i\left(-\frac{z}{2}+2 a(1-s)\right)\right] \Phi(z ; a, 1-s)\right] .
\end{aligned}
$$

Taking $a=1$, we have

$$
e^{2 \pi i s} \Phi(1-z ; s, 1)=(2 \pi)^{-z} \Gamma(z)\left[e^{\frac{\pi i}{2} z} \zeta(z, s)+e^{-\frac{\pi i}{2} z} \zeta(z, 1-s)\right]
$$

\section{References}

[1] L. Jacobs, Phys. Rev. D10 (1974) 3956;

D.J. Harrington and A. Yildiz, Phys. Rev. D11 (1975) 779; 
H. Wada, Nuovo Cimento Lett. 11 (1974) 697;

W. Dittrich and B.G. Englert, Nucl. Phys. B179 (1981) 85;

R.F. Dashen, S.-K. Ma and R. Rajaraman, Phys. Rev. D11 (1975) 1499.

[2] D.J. Harrington, S.Y. Park and A. Yildiz, Phys. Rev. D11 (1975) 1472.

[3] S. Kawati, A. Konisi and H. Miyata, Phys. Rev. D28 (1983) 1537.

[4] S.P. Klevansky and R.H. Lemmer, Phys. Rev. D39 (1989) 3478.

[5] K.G. Klimenko, Theor. Math. Phys. 89 (1992) 1161.

[6] K.G. Klimenko, Theor. Math. Phys. 89 (1992) 1287.

[7] K.G. Klimenko, Z. Phys. C54 (1992) 323.

[8] I.V. Krive and S.A. Naftulin, Phys. Rev. D46 (1992) 2737.

[9] A.Yu. Babansky, E.V. Gorbar and G.V. Shchepanyuk, hep-th/9705218.

[10] C.N.Leung, Y.J.Ng and A.W. Ackley, Phys. Rev. D54 (1996) 4181;

D.S.Lee, C.N.Leung and Y.J.Ng, Phys. Rev. D55 (1997) 6504.

[11] V.P. Gusynin, V.A. Miransky and I.A. Shovkovy, Phys. Rev. D52 (1995) 4718.

[12] V.P. Gusynin, V.A. Miransky and I.A. Shovkovy, Nucl. Phys. B462 (1996) 249; Phys. Rev. Lett. 73 (1994) 3499; V.P. Gusynin and I.A. Shovkovy, Phys. Rev. D56 (1997) 5251.

[13] V. Elias, D.G.C. McKeon, V.A. Miransky and I.A. Shovkovy, Phys. Rev. D54 (1996) 7884 .

[14] S.P. Klevansky, Rev. Mod. Phys. 64 (1992) 649.

[15] T. Inagaki, S. Mukaigawa, T. Muta, Phys. Rev. D52 (1995) 4267;

E. Elizalde, S. Leseduarte, S.D. Odintsov and Yu.I. Shilnov, Phys. Rev. D53 (1996) 1917. 
[16] D.M. Gitman, S.D. Odintsov and Yu.I. Shil'nov, Phys. Rev. D54 (1996) 2968;

B. Geyer, L.N. Granda and S.D. Odintsov, Mod. Phys. Lett. A11 (1996) 2053;

G. Miele and P. Vitale, Nucl. Phys. B494 (1997) 365.

[17] S. Kanemura and H.-T. Sato, Mod. Phys. Lett. A10 (1995) 1777; ibid. A11 (1996) 785.

[18] T.F. Treml, Phys. Rev. D39 (1989) 679;

U. Wolff, Phys. Lett. B157 (1985) 303;

B. Rosenstein, B.J. Warr and S.H. Park, Phys. Rep. 205 (1991) 59.

[19] T. Inagaki, T. Kouno and T. Muta, Int. J. Mod. Phys. A10 (1995) 2241.

[20] A. Chodos and H. Minakata, Phys. Lett. A191 (1994) 39.

[21] A. Chodos and H. Minakata, Nucl. Phys. B490 (1997) 687.

[22] M.J. Strassler, Nucl. Phys. B385 (1992) 145.

[23] M.G. Schmidt and C. Schubert, Phys. Lett. B318 (1993) 438;

M. Reuter, M.G. Schmidt and C. Schubert, Ann. Phys. 259 (1997) 313.

[24] J.O. Andersen and T. Haugset, cond-mat/9410084.

[25] M. Stone, Phys. Rev. D14 (1976) 3568.

[26] J. Schwinger, Phys. Rev. 82 (1951) 664.

[27] D. Cangemi and G. Dunne, Ann. Phys. 249 (1996) 582.

[28] H.-T. Sato, and H. Tochimura, Mod. Phys. Lett. A11 (1996) 3091.

[29] I.S. Gradshteyn and I.M. Ryzhik, Table of Integrals, Series and Products (Academic Press). 